\title{
Multiperiod structure of electroweak phase transition in the 3-3-1-1 model
}

\author{
V. Q. Phong, ${ }^{1, \dagger}$ N. T. Tuong, ${ }^{1, \hbar}$ N. C. Thao, ${ }^{2,3, \S}$ and H. N. Long ${ }^{4,5, *}$ \\ ${ }^{1}$ Department of Theoretical Physics, VNUHCM-University of Science, Ho Chi Minh City 700000, Vietnam \\ ${ }^{2}$ Graduate University of Science and Technology, Vietnam Academy of Science and Technology, \\ 18 Hoang Quoc Viet, Cau Giay, Hanoi 100000, Vietnam \\ ${ }^{3}$ Institute of Physics, Vietnam Academy of Science and Technology, \\ 10 Dao Tan, Ba Dinh, Hanoi 100000, Vietnam \\ ${ }^{4}$ Theoretical Particle Physics and Cosmology Research Group, Advanced Institute for Materials Science, \\ Ton Duc Thang University, Ho Chi Minh City 700000, Vietnam \\ ${ }^{5}$ Faculty of Applied Sciences, Ton Duc Thang University, Ho Chi Minh City 700000, Vietnam
}

(Received 29 May 2018; published 25 January 2019)

\begin{abstract}
The electroweak phase transition (EWPT) is considered in the framework of 3-3-1-1 model for dark matter. The phase structure within three or two periods is approximated for the theory with many vacuum expectation values (VEVs) at $\mathrm{TeV}$ and Electroweak scales. In the mentioned model, there are two pictures. The first picture containing two periods of EWPT, has a transition $S U(3) \rightarrow S U(2)$ at $6 \mathrm{TeV}$ scale and another is $S U(2) \rightarrow U(1)$ transition which is the like-standard model EWPT. The second picture is an EWPT structure containing three periods, in which two first periods are similar to those of the first picture and another one is the symmetry breaking process of $U(1)_{N}$ subgroup. Our study leads to the conclusion that EWPTs are the first order phase transitions when new bosons are triggers and their masses are within range of some TeVs. Especially, in two pictures, the maximum strength of the $S U(2) \rightarrow U(1)$ phase transition is equal to 2.12 so this EWPT is not strong. Moreover, neutral fermions, which are candidates for dark matter and obey the Fermi-Dirac distribution, can be a negative trigger for EWPT. However, they do not make lose the first-order EWPT at TeV scale. Furthermore, in order to be the strong first-order EWPT at $\mathrm{TeV}$ scale, the symmetry breaking processes must produce more bosons than fermions or the mass of bosons must be much larger than that of fermions.
\end{abstract}

DOI: 10.1103/PhysRevD.99.015035

\section{INTRODUCTION}

The EWPT is another view of spontaneous symmetry breaking in theoretical particle physics. The latter is a transition of the Higgs field with vanishing VEV to a nonzero one. The EWPT plays an important role at early stage of expanding universe; and its issue is also related to hot topics such as dark matter (DM) or dark energy. From a micro viewpoint and within the current limits, candidate for DM may be a heavy particle. If we accept the symmetrybreaking mechanism as an universal mechanism, then mass of the DM candidate must also be generated through a

\footnotetext{
*Corresponding author.

hoangngoclong@tdtu.edu.vn

†qphong@hcmus.edu.vn

*nttuong.phys@gmail.com

§ncthao@grad.iop.vast.ac.vn

Published by the American Physical Society under the terms of the Creative Commons Attribution 4.0 International license. Further distribution of this work must maintain attribution to the author(s) and the published article's title, journal citation, and DOI. Funded by SCOAP.
}

phase transition process. Moreover, if the mass of the DM candidate is very large so the phase transition process must take place before the EWPT of the standard model (SM) and must also follow the gradually decreasing temperature structure of the universe.

As in the SM, the EWPT process has only one phase at the energy level around $200 \mathrm{GeV}$. This process is accompanied by mass generation of particles. However, at present, the existence of heavy particles is possible only at energy scale larger than $200 \mathrm{GeV}$. Therefore, the production of these heavy particles interacting with the SM ones must also be considered.

At present, the mechanism of symmetry-breaking is believed to be accurate, but the Higgs potential is not exactly determined because its form is model dependent.

The EWPT consists of an important question of phase transition which must be a strongly first-order phase one. This is the third Sakharov condition being deviation from thermal equilibrium Ref. [1]. The mentioned condition together with $\mathrm{B}, \mathrm{C}, C P$ violations leads to solution of the baryon asymmetry of universe (BAU). The $\mathrm{B}, \mathrm{C}$, and $C P$ violations can be seen throughout the sphaleron rate and the 
CKM-matrix in models Ref. [2] or other $C P$ violation sources as neutrino mixing or heavy extra neutrinos via adding see-saw mechanism Ref. [3].

At present, the EWPT is considered at a one-loop level, particularly, in beyond the standard model. A new trend nowadays is multiphase calculations in multi-Higgs scalar potential.

In order to consider the EWPT, we must build the hightemperature effective potential which is usually in the following form

$$
V_{\text {eff }}=D \cdot\left(T^{2}-T_{0}^{2}\right) v^{2}-E \cdot T v^{3}+\frac{\lambda_{T}}{4} v^{4},
$$

where $v$ is the VEV of Higgs boson. The first order EWPT binds that the strength of phase transition should be larger than the unit $\left(S=\frac{v_{c}}{T_{c}} \geq 1\right.$, where $v_{c}$ is VEV of Higgs field at a critical temperature $T_{c}$ ).

The effective potential $V_{\text {eff }}$ in Eq. (1) is a function of temperature and VEVs. It can have one or two minimums when the temperature goes down. At $T_{c}$, the two minimums are separated by a potential barrier, the VEV of Higgs field crosses over from vanishing VEV to a nonzero VEV. This transition is called the first order phase transition and it can cause large deviations from thermal equilibrium.

The EWPT has been calculated in the SM Ref. [2] and in some extended models Refs. [4-19]. We recall that DM, heavy particles, and neutrino oscillations can be triggers of the EWPT, Ref. [20]. Most studies of the EWPT are performed in the framework of the Landau gauge. However gauge also made contributions in EWPT as done in Ref. [19]. We recall that in some extended models, Higgs sector consists multivacuum structure of which the classical example is the two Higgs doublet model and new models with $S U(5)$ and $S U(6)$ groups Ref. [21] or the $S U(3)_{C} \otimes$ $S U(2)_{L} \otimes U(1)_{Y}\left(\otimes U(1)_{X}\right)$ model as a symmetry of division algebraic ladder operators Ref. [22]. This additional Higgs structure can be a new source to answer the BAU puzzles.

Another example of multivacuum structure belongs to the models based on $S U(3)_{C} \otimes S U(3)_{L} \otimes U(1)_{X}$ group Refs. [23,24] called 3-3-1 models for short. There exist two main versions of the 3-3-1 models: the minimal, Ref. [23], and another with right-handed neutrinos, Ref. [24]. To provide an explanation for the observed pattern of SM fermion masses and mixings, various 3-3-1 models with flavor symmetries and radiative seesaw mechanisms have been proposed in the literature. ${ }^{1}$ However some of them involve nonrenormalizable interactions. In addition the 3-3-1 models do not give completely desired answer on the DM issue. In the recently proposed 3-3-1-1 model

\footnotetext{
${ }^{1}$ With the help of discrete $Z_{N}$ symmetries, the 3-3-1 model with $\beta=\frac{1}{\sqrt{3}}$ can provide solutions of neutrino mass and mixing, $\mathrm{DM}$ and inflation Refs. [25,26].
}

Ref. [27] based on $S U(3)_{C} \otimes S U(3)_{L} \otimes U(1)_{X} \otimes U(1)_{N}$ group has a good advantage in explaining DM. Phenomena of this model such as DM, inflation, leptogenesis, neutrino mass, kinetic mixing effect, and $B-L$ asymmetry, have been studied in Refs. [28-32]. The 3-3-1-1 model has three Higgs triplets to generate masses of fermions and the mass of new heavy particle with masses around some TeVs. This model fits with candidates for DM. The presence of the above mentioned particles might also lead to interesting consequences such as the baryon asymmetry or EWPT which is a subject of this study.

This article is organized as follows. In Sec. II, the matter fields and Higgs bosons in the 3-3-1-1 model are briefly reviewed. In Sec. III, the effective potential having the contribution from heavy bosons a function of temperature and VEVs is derived. In Sec. IV, we analyze in detail the structure of phase transition, find the first order phase transition, and show constraints on mass of charged Higgs boson in the case without neutral fermions. In Sec. V, we discuss the role of neutral fermions in the EWPT problem. Finally, we summarize and make outlooks in Sec. VI.

\section{BRIEF REVIEW OF THE 3-3-1-1 MODEL}

It is well known that the SM must be extended and most versions of the BSM contain heavy particles. Within the latter existence, unexplained problems can be caused. The heavy particles may be a candidate for DM, or just new ones. The 3-3-1-1 model has many new particles inserting in the multiplets of the gauge group $S U(3)_{C} \otimes S U(3)_{L} \otimes$ $U(1)_{X} \otimes U(1)_{N}$, where the latter is the subgroup associated with the conservation of $B-L$ number [27-31].

To keep the model being anomaly free, the fermion content has to have an equal number of the $S U(3)_{L}$ triplets and antitriplets as follows [27]

$$
\begin{aligned}
\psi_{a L} & =\left(\nu_{a L}, e_{a L},\left(N_{a R}\right)^{c}\right)^{T} \sim\left(1,3,-\frac{1}{3},-\frac{2}{3}\right), \\
e_{a R} & \sim(1,1,-1,-1), \quad \nu_{a R} \sim(1,1,0,-1), \\
Q_{\alpha L} & =\left(d_{\alpha L},-u_{\alpha L}, D_{\alpha L}\right)^{T} \sim\left(3,3^{*}, 0,0\right), \\
Q_{3 L} & =\left(u_{3 L}, d_{3 L}, U_{L}\right)^{T} \sim(3,3,1 / 3,2 / 3), \\
u_{a R} & \sim\left(3,1, \frac{2}{3}, \frac{1}{3}\right), \quad d_{a R} \sim\left(3,1,-\frac{1}{3}, \frac{1}{3}\right), \\
U_{R} & \sim\left(3,1, \frac{2}{3}, \frac{4}{3}\right), \quad D_{\alpha R} \sim\left(3,1,-\frac{1}{3},-\frac{2}{3}\right),
\end{aligned}
$$

where $a=1,2,3$ and $\alpha=1,2$ are family indices. $N_{a R}$ is neutral fermions playing a role of candidates for DM. In (2), the numbers in bracket associated with multiplet correspond to number of members in the $S U(3)_{C}$, $S U(3)_{L}$ assignment, its $X$ and $N$ charges, respectively.

The Higgs sector of the model under consideration contains three scalar triplets and one singlet as follows 
TABLE I. Nonzero lepton number $L$ of fields in the 3-3-1-1 model.

\begin{tabular}{lcccccccccc}
\hline \hline Particle & $\nu$ & $e$ & $N$ & $U$ & $D$ & $\eta_{3}$ & $\rho_{3}$ & $\chi_{1}$ & $\chi_{2}$ & $\phi$ \\
\hline$L$ & 1 & 1 & 0 & -1 & 1 & -1 & -1 & 1 & 1 & -2 \\
\hline \hline
\end{tabular}

$$
\begin{aligned}
& \eta=\left(\eta_{1}^{0}, \eta_{2}^{-}, \eta_{3}^{0}\right)^{T} \sim(1,3,-1 / 3,1 / 3), \\
& \chi=\left(\chi_{1}^{0}, \chi_{2}^{-}, \chi_{3}^{0}\right)^{T} \sim(1,3,-1 / 3,-2 / 3),
\end{aligned}
$$

$\rho=\left(\rho_{1}^{+}, \rho_{2}^{0}, \rho_{3}^{+}\right)^{T} \sim(1,3,2 / 3,1 / 3), \quad \phi \sim(1,1,0,2)$.

Note that in (2), the lepton and antilepton lie in the same triplet. Hence, lepton number is not conserved and it should be replaced with new conserved one $\mathcal{L}$ [33]. Assuming the bottom element in lepton triplet $\left(N_{a R}\right)$ without lepton number, ones have [27]

$$
B-L=-\frac{2}{\sqrt{3}} T_{8}+N
$$

Note that in this model, not only leptons but also some scalar fields carry lepton number as seen in Table I.

From Table I, we see that elements at the bottom of $\eta$ and $\rho$ triplets carry lepton number -1 , while the elements standing in two first rows of $\chi$ triplet have the opposite one +1 .

To generate masses for fermions, it is enough that only neutral scalars without lepton number develop VEV as follows

$$
\begin{aligned}
& \langle\eta\rangle=\left(\frac{u}{\sqrt{2}}, 0,\right)^{T}, \quad\langle\chi\rangle=\left(0,0, \frac{\omega}{\sqrt{2}}\right)^{T}, \\
& \langle\rho\rangle=\left(0, \frac{v}{\sqrt{2}}, 0\right)^{T} .
\end{aligned}
$$

For the future presentation, let us remind that in the model under consideration, the covariant derivative is defined as

$$
D_{\mu}=\partial_{\mu}-i g_{s} t_{i} G_{i \mu}-i g T_{i} A_{i \mu}-i g_{X} X B_{\mu}-i g_{N} N C_{\mu},
$$

where $G_{i \mu \nu}, A_{i \mu \nu}, B_{\mu \nu}, C_{\mu \nu}$ and $g_{s}, g, g_{X}, g_{N}$ correspond gauge fields and couplings of $S U(3)_{C}, S U(3)_{L}, U(1)_{X}$ and $U(1)_{N}$ groups, respectively.

The Yukawa couplings are given as

$$
\begin{aligned}
\mathcal{L}_{\text {Yukawa }}= & h_{a b}^{e} \bar{\psi}_{a L} \rho e_{b R}+h_{a b}^{\nu} \bar{\psi}_{a L} \eta \nu_{b R}+h_{a b}^{\nu} \bar{\nu}_{a R}^{c} \nu_{b R} \phi \\
& +h^{U} \bar{Q}_{3 L} \chi U_{R}+h_{\alpha \beta}^{D} \bar{Q}_{\alpha L} \chi^{*} D_{\beta R} \\
& +h_{a}^{u} \bar{Q}_{3 L} \eta u_{a R}+h_{a}^{d} \bar{Q}_{3 L} \rho d_{a R} \\
& +h_{a b}^{d} \bar{Q}_{a L} \eta^{*} d_{b R}+h_{a b}^{u} \bar{Q}_{a L} \rho^{*} u_{b R}+\text { H.c. }
\end{aligned}
$$

From Eq. (8), it follows that masses of the top and bottom quarks as follows

$$
m_{t}=\frac{h_{t} u}{\sqrt{2}}, \quad m_{b}=\frac{h_{b} v}{\sqrt{2}},
$$

while masses of the exotic quarks are determined as

$$
m_{U}=\frac{\omega}{\sqrt{2}} h^{U} ; \quad m_{D_{1}}=\frac{\omega}{\sqrt{2}} h_{11}^{D} ; \quad m_{D_{2}}=\frac{\omega}{\sqrt{2}} h_{22}^{D} .
$$

The Higgs fields are expanded around the VEVs as follows

$$
\begin{aligned}
& \eta=\langle\eta\rangle+\eta^{\prime}, \eta^{\prime}=\left(\frac{S_{\eta}+i A_{\eta}}{\sqrt{2}}, \eta^{-}, \frac{S_{\eta}^{\prime}+i A_{\eta}^{\prime}}{\sqrt{2}}\right), \\
& \rho=\langle\rho\rangle+\rho^{\prime}, \rho^{\prime}=\left(\rho^{+}, \frac{S_{\rho}+i A_{\rho}}{\sqrt{2}}, \rho^{\prime+}\right), \\
& \chi=\langle\chi\rangle+\chi^{\prime}, \chi^{\prime}=+\left(\frac{S_{\chi}+i A_{\chi}}{\sqrt{2}}, \chi^{-}, \frac{S_{\chi}^{\prime}+i A_{\chi}^{\prime}}{\sqrt{2}}\right), \\
& \phi=\langle\phi\rangle+\phi^{\prime}=\frac{\Lambda}{\sqrt{2}}+\frac{S_{4}+i A_{4}}{\sqrt{2}} .
\end{aligned}
$$

It is mentioned that the values $u$ and $v$ provide masses for all fermions and gauge bosons in the SM, while $\omega$ gives masses for the extra heavy quarks and gauge bosons. The value $\Lambda$ plays the role for the $U(1)_{N}$ breaking at high scale; and in some cases, it is larger than $\omega$.

The scalar potential for Higgs fields is a function of eighteen parameters

$$
\begin{aligned}
V(\rho, \eta, \chi, \phi)= & \mu_{1}^{2} \rho^{\dagger} \rho+\mu_{2}^{2} \chi^{\dagger} \chi+\mu_{3}^{2} \eta^{\dagger} \eta+\lambda_{1}\left(\rho^{\dagger} \rho\right)^{2}+\lambda_{2}\left(\chi^{\dagger} \chi\right)^{2}+\lambda_{3}\left(\eta^{\dagger} \eta\right)^{2} \\
& +\lambda_{4}\left(\rho^{\dagger} \rho\right)\left(\chi^{\dagger} \chi\right)+\lambda_{5}\left(\rho^{\dagger} \rho\right)\left(\eta^{\dagger} \eta\right)+\lambda_{6}\left(\chi^{\dagger} \chi\right)\left(\eta^{\dagger} \eta\right) \\
& \left.+\lambda_{7}\left(\rho^{\dagger} \chi\right)\left(\chi^{\dagger} \rho\right)+\lambda_{8}\left(\rho^{\dagger} \eta\right)\left(\eta^{\dagger} \rho\right)+\lambda_{9}\left(\chi^{\dagger} \eta\right)\left(\eta^{\dagger} \chi\right)+f \varepsilon^{m n p} \eta_{m} \rho_{n} \chi_{p}+\text { H.c. }\right) \\
& +\mu^{2} \phi^{\dagger} \phi+\lambda\left(\phi^{\dagger} \phi\right)^{2}+\lambda_{10}\left(\phi^{\dagger} \phi\right)\left(\rho^{\dagger} \rho\right)+\lambda_{11}\left(\phi^{\dagger} \phi\right)\left(\chi^{\dagger} \chi\right)+\lambda_{12}\left(\phi^{\dagger} \phi\right)\left(\eta^{\dagger} \eta\right) .
\end{aligned}
$$

When constructing this Higgs potential, triple scalar selfinteractions needs to be limited because it forces us to introduce a $f$ parameter ( $f$ has a mass dimension the same as $\omega$ ) that can like an interrupt factor for these interactions.
In addition, $f$ can be replaced by one Higgs field or another interaction among three Higgs fields. Thus, the mentioned interaction will become a fourth- or sixth-order coupling. We often do not consider high-order interactions (because 
these high-order interactions may be difficult to renormalization. However they may be related to other hypothetical offending processes). Therefore we can ignore $f$ in this article though it may have a different role in other problems. For detailed analysis of the Higgs sector in the model under consideration, the reader is referred to Ref. [27].

In this particular model, the mass of scalar boson depends on not only VEVs, $\mu_{1,2,3}$ and $\lambda_{i}, \quad i=$ $1,2,3, \ldots, 12$ but also $f$ parameter. Note that $f$ increases the mass of bosons [27]. Returning to our work, in order to limit the parameter number, as above mentioned, we will ignore $f$ hereafter.

\section{A. Higgs boson masses}

Substituting Eq. (9) into Eq. (10) yields

$$
\begin{aligned}
V(\rho, \eta, \chi, \phi)= & V_{0}+V_{1}+\sum_{i=\rho, \eta, \chi}\left(V_{S_{i}}+V_{A_{i}}+V_{i}\right) \\
& +V_{S_{4}}+V_{A_{4}}+\text { Interaction terms }
\end{aligned}
$$

where $V_{0}$ and $V_{1}$ are the minimum interaction term being independent of scalar fields and linear dependent on fields, respectively:

$$
\begin{aligned}
V_{0}= & \frac{\lambda^{2} \Lambda^{4}}{4}+\frac{1}{4} \lambda_{11} \Lambda^{2} \omega^{2}+\frac{\lambda_{2} \omega^{4}}{4}+\frac{\Lambda^{2} \mu^{2}}{2}+\frac{1}{2} \mu_{2}^{2} \omega^{2} \\
& +\frac{\lambda_{3} u^{4}}{4}+\frac{1}{4} \lambda_{12} \Lambda^{2} u^{2}+\frac{1}{4} \lambda_{6} u^{2} \omega^{2} \\
& +\frac{1}{2} \mu_{3}^{2} u^{2}+\frac{1}{4} \lambda_{5} u^{2} v^{2}+\frac{\lambda_{1} v^{4}}{4}+\frac{1}{4} \lambda_{10} \Lambda^{2} v^{2} \\
& +\frac{1}{4} \lambda_{4} v^{2} \omega^{2}+\frac{1}{2} \mu_{1}^{2} v^{2}
\end{aligned}
$$

$$
\begin{aligned}
V_{1}= & S_{\eta}\left[u \mu_{3}^{2}+\lambda_{3} u^{3}+\frac{1}{2} \lambda_{5} u v^{2}+\frac{1}{2} \lambda_{6} u \omega^{2}+\frac{1}{2} \lambda_{12} u \Lambda^{2}\right] \\
& +S_{\rho}\left[v \mu_{1}^{2}+\lambda_{1} v^{3}+\frac{1}{2} \lambda_{4} v \omega^{2}+\frac{1}{2} \lambda_{5} u^{2} v+\frac{1}{2} \lambda_{10} v \Lambda^{2}\right] \\
& +S_{\chi}\left[\omega \mu_{2}^{2}+\lambda_{2} \omega^{3}+\frac{1}{2} \lambda_{4} \omega v^{2}+\frac{1}{2} \lambda_{6} u^{2} \omega+\frac{1}{2} \lambda_{11} \omega \Lambda^{2}\right] \\
& +S_{4}\left[\Lambda \mu^{2}+\lambda \Lambda^{3}+\frac{1}{2} \lambda_{10} v^{2} \Lambda+\frac{1}{2} \lambda_{11} \Lambda \omega^{2}+\frac{1}{2} \lambda_{12} \Lambda u^{2}\right] .
\end{aligned}
$$

Hence, the potential minimization conditions are obtained by

$$
\begin{gathered}
u\left(\lambda_{12} \Lambda^{2}+\lambda_{6} \omega^{2}+2 \mu_{3}^{2}+2 \lambda_{3} u^{2}+\lambda_{5} v^{2}\right)=0, \\
\omega\left(\lambda_{11} \Lambda^{2}+2 \lambda_{2} \omega^{2}+2 \mu_{2}^{2}+\lambda_{6} u^{2}+\lambda_{4} v^{2}\right)=0,
\end{gathered}
$$

$$
v\left(\lambda_{10} \Lambda^{2}+\lambda_{4} \omega^{2}+2 \mu_{1}^{2}+\lambda_{5} u^{2}+2 \lambda_{1} v^{2}\right)=0,
$$

From (11) we get the part for charged Higgs bosons

$$
\begin{aligned}
V_{\eta} & =\lambda_{3}\left(\eta^{+} \eta^{-}\right)^{2}+\left(\frac{\Lambda^{2} \lambda_{12}}{2}+\frac{\lambda_{6} \omega^{2}}{2}+\mu_{3}^{2}+\lambda_{3} u^{2}+\frac{\lambda_{5} v^{2}}{2}+\frac{\lambda_{8} v^{2}}{2}\right) \eta^{+} \eta^{-}+\frac{1}{2} \lambda_{8} u v \eta^{\dagger} \rho^{-} \\
& =\lambda_{3}\left(\eta^{+} \eta^{-}\right)^{2}+\left(\frac{\lambda_{8} v^{2}}{2}\right) \eta^{+} \eta^{-}+\frac{1}{2} \lambda_{8} u v \eta^{\dagger} \rho^{-}, \\
V_{\chi} & =\lambda_{2}\left(\chi^{+} \chi^{-}\right)^{2}+\left(\frac{\Lambda^{2} \lambda_{11}}{2}+\lambda_{2} \omega^{2}+\mu_{2}^{2}+\frac{\lambda_{6} u^{2}}{2}+\frac{\lambda_{7} v^{2}}{2}+\frac{\lambda_{4} v^{2}}{2}\right) \chi^{+} \chi^{-}+\frac{1}{2} \lambda_{7} v \omega \chi^{-} \rho^{\prime \dagger} \\
& =\lambda_{2}\left(\chi^{+} \chi^{-}\right)^{2}+\left(\frac{\lambda_{7} v^{2}}{2}\right) \chi^{+} \chi^{-}+\frac{1}{2} \lambda_{7} v \omega \chi^{-} \rho^{\prime \dagger}, \\
V_{\rho} & =\lambda_{1}\left(\rho^{+} \rho^{-}\right)^{2}+\left(\frac{\Lambda^{2} \lambda_{10}}{2}+\frac{\lambda_{4} \omega^{2}}{2}+\mu_{1}^{2}+\frac{\lambda_{5} u^{2}}{2}+\frac{\lambda_{8} u^{2}}{2}+\lambda_{1} v^{2}\right) \rho^{+} \rho^{-}+\frac{1}{2} \lambda_{8} u v \eta^{-} \rho^{\dagger} \\
& =\lambda_{1}\left(\rho^{+} \rho^{-}\right)^{2}+\left(\frac{\lambda_{8} u^{2}}{2}\right) \rho^{+} \rho^{-}+\frac{1}{2} \lambda_{8} u v \eta^{-} \rho^{\dagger}, \\
V_{\rho^{\prime}} & =\lambda_{1}\left(\rho^{\prime+} \rho^{\prime-}\right)^{2}+\left(\frac{\Lambda^{2} \lambda_{10}}{2}+\frac{\lambda_{7} \omega^{2}}{2}+\frac{\lambda_{4} \omega^{2}}{2}+\mu_{1}^{2}+\frac{\lambda_{5} u^{2}}{2}+\lambda_{1} v^{2}\right) \rho^{\prime+} \rho^{\prime-}+\frac{1}{2} \lambda_{7} v \omega \chi^{\dagger} \rho^{\prime-} \\
& =\lambda_{1}\left(\rho^{\prime+} \rho^{\prime-}\right)^{2}+\left(\frac{\lambda_{7} \omega^{2}}{2}\right) \rho^{\prime+} \rho^{\prime-}+\frac{1}{2} \lambda_{7} v \omega \chi^{\dagger} \rho^{\prime-} .
\end{aligned}
$$


From the above equations, after some manipulations, the mass terms of charged Higgs bosons are given by

$$
\begin{aligned}
V_{\text {Higgs }}^{\text {mass }}= & \left(\frac{\lambda_{8} v^{2}}{2}\right) \eta^{+} \eta^{-}+\frac{1}{2} \lambda_{8} u v \eta^{\dagger} \rho^{-}+\left(\frac{\lambda_{8} u^{2}}{2}\right) \rho^{+} \rho^{-}+\frac{1}{2} \lambda_{8} u v \eta^{-} \rho^{\dagger}+\left(\frac{\lambda_{7} v^{2}}{2}\right) \chi^{+} \chi^{-}+\frac{1}{2} \lambda_{7} v \omega \chi^{-} \rho^{\prime \dagger} \\
& +\left(\frac{\lambda_{7} \omega^{2}}{2}\right) \rho^{\prime+} \rho^{\prime-}+\frac{1}{2} \lambda_{7} v \omega \chi^{\dagger} \rho^{\prime-} \\
= & \frac{u^{2}+v^{2}}{2} \lambda_{8}\left(\frac{v \eta^{+}+u \rho^{+}}{\sqrt{u^{2}+v^{2}}}\right)\left(\frac{v \eta^{-}+u \rho^{-}}{\sqrt{u^{2}+v^{2}}}\right)+\frac{\omega^{2}+v^{2}}{2} \lambda_{7}\left(\frac{v \eta^{+}+\omega \rho^{+}}{\sqrt{\omega^{2}+v^{2}}}\right)\left(\frac{v \eta^{-}+\omega \rho^{-}}{\sqrt{\omega^{2}+v^{2}}}\right) \\
= & \frac{u^{2}+v^{2}}{2} \lambda_{8} H_{1}^{+} H_{1}^{-}+\frac{\omega^{2}+v^{2}}{2} \lambda_{7} H_{2}^{+} H_{2}^{-} \\
= & m_{H_{1}}^{2} H_{1}^{+} H_{1}^{-}+m_{H_{2}}^{2} H_{2}^{+} H_{2}^{-},
\end{aligned}
$$

where

$$
H_{1}^{ \pm}=\frac{v \eta^{ \pm}+u \rho^{ \pm}}{\sqrt{u^{2}+v^{2}}} ; \quad H_{2}^{ \pm}=\frac{v \eta^{ \pm}+\omega \rho^{ \pm}}{\sqrt{\omega^{2}+v^{2}}} ; \quad m_{H_{1}}^{2}=\frac{u^{2}+v^{2}}{2} \lambda_{8} ; \quad m_{H_{2}}^{2}=\frac{\omega^{2}+v^{2}}{2} \lambda_{7}
$$

Similarly, the part of neutral Higgs bosons is given by:

$$
\begin{aligned}
& V_{A_{4}}=\frac{\lambda}{4} A_{4}^{4}+\left(\frac{1}{2} \lambda \Lambda^{2}+\frac{1}{4} \omega^{2} \lambda_{11}+\frac{\mu^{2}}{2}+\frac{\lambda_{12} u^{2}}{4}+\frac{\lambda_{10} v^{2}}{4}\right) A_{4}^{2}=\frac{\lambda}{4} A_{4}^{4}, \\
& V_{S_{4}}=\frac{\lambda}{4} S_{4}^{4}+\left(\frac{3}{2} \lambda \Lambda^{2}+\frac{1}{4} \omega^{2} \lambda_{11}+\frac{\mu^{2}}{2}+\frac{\lambda_{12} u^{2}}{4}+\frac{\lambda_{10} v^{2}}{4}\right) S_{4}^{2}=\frac{\lambda}{4} S_{4}^{4}+\lambda \Lambda^{2} S_{4}^{2}, \\
& V_{A_{\eta}}=\frac{\lambda_{3}}{4} A_{\eta}^{4}+\left(\frac{\Lambda^{2} \lambda_{12}}{4}+\frac{\lambda_{6} \omega^{2}}{4}+\frac{\mu_{3}^{2}}{2}+\frac{\lambda_{3} u^{2}}{2}+\frac{\lambda_{5} v^{2}}{4}\right) A_{\eta}^{2}=\frac{\lambda_{3}}{4} A_{\eta}^{4} \\
& V_{A_{\eta}^{\prime}}=\frac{\lambda_{3}}{4} A_{\eta}^{\prime 4}+\left(\frac{\Lambda^{2} \lambda_{12}}{4}+\frac{\lambda_{6} \omega^{2}}{4}+\frac{\lambda_{9} \omega^{2}}{4}+\frac{\mu_{3}^{2}}{2}+\frac{\lambda_{3} u^{2}}{2}+\frac{\lambda_{5} v^{2}}{4}\right) A_{\eta}^{\prime 2}=\frac{\lambda_{3}}{4} A_{\eta}^{\prime 4}+\frac{\lambda_{9} \omega^{2}}{4} A_{\eta}^{\prime 2}, \\
& V_{A_{\chi}}=\frac{\lambda_{2}}{4} A_{\chi}^{4}+\left(\frac{\Lambda^{2} \lambda_{11}}{4}+\frac{\lambda_{2} \omega^{2}}{2}+\frac{\mu_{2}^{2}}{2}+\frac{\lambda_{6} u^{2}}{4}+\frac{\lambda_{9} u^{2}}{4}+\frac{\lambda_{4} v^{2}}{4}\right) A_{\chi}^{2}=\frac{\lambda_{2}}{4} A_{\chi}^{4}+\frac{\lambda_{9} u^{2}}{4} A_{\chi}^{2}, \\
& V_{A_{\chi}^{\prime}}=\frac{\lambda_{2}}{4} A_{\chi}^{\prime 4}+\left(\frac{\Lambda^{2} \lambda_{11}}{4}+\frac{\lambda_{2} \omega^{2}}{2}+\frac{\mu_{2}^{2}}{2}+\frac{\lambda_{6} u^{2}}{4}+\frac{\lambda_{4} v^{2}}{4}\right) A_{\chi}^{\prime 2}=\frac{\lambda_{2}}{4} A_{\chi}^{\prime 4}, \\
& V_{A_{\rho}}=\frac{\lambda_{1}}{4} A_{\rho}^{4}+\left(\frac{\Lambda^{2} \lambda_{10}}{4}+\frac{\lambda_{4} \omega^{2}}{4}+\frac{\mu_{1}^{2}}{2}+\frac{\lambda_{5} u^{2}}{4}+\frac{\lambda_{1} v^{2}}{2}\right) A_{\rho}^{2}=\frac{\lambda_{1}}{4} A_{\rho}^{4},=\frac{\lambda_{3}}{4} S_{\eta}^{4}+u \lambda_{3} S_{\eta}^{3}+\lambda_{3} u^{2} S_{\eta}^{2}, \\
& V_{S_{\chi}^{\prime}}=\frac{\lambda_{2}}{4} S_{\chi}^{\prime 4}+\omega \lambda_{2} S_{\chi}^{\prime 3}+\left(\frac{\Lambda^{2} \lambda_{11}}{4}+\frac{3 \lambda_{2} \omega^{2}}{2}+\frac{\mu_{2}^{2}}{2}+\frac{\lambda_{6} u^{2}}{4}+\frac{\lambda_{4} v^{2}}{4}\right) S_{\chi}^{\prime 2}=\frac{\lambda_{2}}{4} S_{\chi}^{\prime 4}+\omega \lambda_{2} S_{\chi}^{\prime 3}+\lambda_{2} \omega^{2} S_{\chi}^{\prime 2}, \\
& V_{S_{\rho}}=\frac{\lambda_{1}}{4} S_{\rho}^{4}+v \lambda_{1} S_{\rho}^{3}+\left(\frac{\Lambda^{2} \lambda_{10}}{4}+\frac{\lambda_{4} \omega^{2}}{4}+\frac{\mu_{1}^{2}}{2}+\frac{\lambda_{5} u^{2}}{4}+\frac{3}{2} \lambda_{1} v^{2}\right) S_{\rho}^{2}=\frac{\lambda_{1}}{4} S_{\rho}^{4}+v \lambda_{1} S_{\rho}^{3}+\lambda_{1} v^{2} S_{\rho}^{2}, \\
& V_{S_{\eta}^{\prime}}=\frac{\lambda_{3}}{4} S_{\eta}^{\prime 4}+\left(\frac{\Lambda^{2} \lambda_{12}}{4}+\frac{\lambda_{6} \omega^{2}}{4}+\frac{\lambda_{9} \omega^{2}}{4}+\frac{\mu_{3}^{2}}{2}+\frac{\lambda_{3} u^{2}}{2}+\frac{\lambda_{5} v^{2}}{4}\right) S_{\eta}^{\prime 2}+\frac{1}{2} \lambda_{9} u \omega S_{\eta}^{\prime} S_{\chi}=\frac{\lambda_{3}}{4} S_{\eta}^{\prime 4}+\frac{\lambda_{9} \omega^{2}}{4} S_{\eta}^{\prime 2}+\frac{1}{2} \lambda_{9} u \omega S_{\eta}^{\prime} S_{\chi}, \\
& V_{S_{\chi}}=\frac{\lambda_{2}}{4} S_{\chi}^{4}+\left(\frac{\Lambda^{2} \lambda_{11}}{4}+\frac{\lambda_{2} \omega^{2}}{2}+\frac{\mu_{2}^{2}}{2}+\frac{\lambda_{6} u^{2}}{4}+\frac{\lambda_{9} u^{2}}{4}+\frac{\lambda_{4} v^{2}}{4}\right) S_{\chi}^{2}=\frac{\lambda_{2}}{4} S_{\chi}^{4}+\frac{\lambda_{9} u^{2}}{4} S_{\chi}^{2} .
\end{aligned}
$$


TABLE II. The neutral Higgs boson masses.

\begin{tabular}{lccccccc}
\hline \hline Neutral Higgs boson & $S_{4}$ & $A^{\prime}{ }_{\eta}$ & $A_{\chi}$ & $S_{\eta}$ & $S_{\chi}^{\prime}$ & $S_{\rho}$ & $H_{3}$ \\
\hline Squared mass & $2 \lambda \Lambda^{2}$ & $\frac{\lambda_{9} \omega^{2}}{2}$ & $\frac{\lambda_{9} u^{2}}{2}$ & $2 \lambda_{3} u^{2}$ & $2 \lambda_{2} \omega^{2}$ & $2 \lambda_{1} v^{2}$ & $\frac{\lambda_{9}\left(u^{2}+\omega^{2}\right)}{2}$ \\
\hline \hline
\end{tabular}

Combination among $S_{\chi}$ and $S_{\eta}^{\prime}$ yields

$$
\begin{aligned}
V_{m}\left(S_{\chi}, S_{\eta}^{\prime}\right) & =\frac{\lambda_{9} \omega^{2}}{4} S_{\eta}^{\prime 2}+\frac{1}{2} \lambda_{9} u \omega S_{\eta}^{\prime} S_{\chi}+\frac{\lambda_{9} u^{2}}{4} S_{\chi}^{2} \\
& =\frac{\lambda_{9}}{4}\left(\omega^{2} S_{\eta}^{\prime 2}+2 u \omega S^{\prime}{ }_{\eta} S_{\chi}+u^{2} S_{\chi}^{2}\right) \\
& =\frac{\lambda_{9}\left(u^{2}+\omega^{2}\right)}{4}\left(\frac{\omega S_{\eta}^{\prime}}{\sqrt{u^{2}+\omega^{2}}}+\frac{u S_{\chi}}{\sqrt{u^{2}+\omega^{2}}}\right)^{2} \\
& =\frac{\lambda_{9}\left(u^{2}+\omega^{2}\right)}{4}\left(H_{3}\right)^{2}=\frac{1}{2} m_{H_{3}}^{2}\left(H_{3}\right)^{2},
\end{aligned}
$$

where physical boson $\mathrm{H}_{3}$ is given by

$H_{3}=\frac{\omega S_{\eta}^{\prime}}{\sqrt{u^{2}+\omega^{2}}}+\frac{u S_{\chi}}{\sqrt{u^{2}+\omega^{2}}}$; with $m_{H_{3}}^{2}=\frac{\lambda_{9}\left(u^{2}+\omega^{2}\right)}{2}$.

The mass of neutral Higgs bosons is presented in Table II.
Remember that the massless Goldstones bosons are: $\mathrm{X}$ $A_{4}, A_{\eta}, A_{\chi}^{\prime}, A_{\rho}$ in neutral scalar sector and two massless combinations orthogonal to the charged Higgs bosons. It is noted that at the limit $f \rightarrow 0$, the results given in [28-30] are consistent with those of this study.

\section{B. Gauge boson sector}

The gauge bosons obtain masses when the scalar fields develop the VEVs. Therefore, their mass Lagrangian is given by

$$
\mathcal{L}_{\text {mass }}^{\text {gauge }}=\sum_{\Phi}\left(D^{\mu}\langle\Phi\rangle\right)^{\dagger}\left(D_{\mu}\langle\Phi\rangle\right)
$$

Substituting the scalar multiplets $\eta, \rho, \chi$, and $\phi$ with their covariant derivative, we obtain

$$
\begin{aligned}
\mathcal{L}_{\text {gauge }}^{\text {mass }}= & \frac{g^{2} u^{2}}{8}\left[\left(A_{3 \mu}+\frac{A_{8 \mu}}{\sqrt{3}}-\frac{2}{3} t_{X} B_{\mu}+\frac{2}{3} t_{N} C_{\mu}\right)^{2}+2 W_{\mu}^{+} W^{-\mu}+2 X_{\mu}^{0 *} X^{0 \mu}\right] \\
& +\frac{g^{2} v^{2}}{8}\left[\left(-A_{3 \mu}+\frac{A_{8 \mu}}{\sqrt{3}}+\frac{4}{3} t_{X} B_{\mu}+\frac{2}{3} t_{N} C_{\mu}\right)^{2}+2 W_{\mu}^{+} W^{-\mu}+2 Y_{\mu}^{+} Y^{-\mu}\right] \\
& +\frac{g^{2} \omega^{2}}{8}\left[\left(-\frac{2 A_{8 \mu}}{\sqrt{3}}-\frac{2}{3} t_{X} B_{\mu}-\frac{4}{3} t_{N} C_{\mu}\right)^{2}+2 Y_{\mu}^{+} Y^{-\mu}+2 X_{\mu}^{0 *} X^{0 \mu}\right]+2 g_{N}^{2} \Lambda^{2} C_{\mu}^{2},
\end{aligned}
$$

where we have denoted $t_{X} \equiv \frac{g_{X}}{g}, t_{N} \equiv \frac{g_{N}}{g}$, and

$$
W_{\mu}^{ \pm}=\frac{A_{1 \mu} \mp i A_{2 \mu}}{\sqrt{2}}, \quad X_{\mu}^{0,0 *}=\frac{A_{4 \mu} \mp i A_{5 \mu}}{\sqrt{2}}, \quad Y_{\mu}^{\mp}=\frac{A_{6 \mu} \mp i A_{7 \mu}}{\sqrt{2}} .
$$

The mass Lagrangian can be rewritten as [28-31]

$$
\mathcal{L}_{\text {mass }}^{\text {gauge }}=\frac{g^{2}}{4}\left(u^{2}+v^{2}\right) W^{+} W^{-}+\frac{g^{2}}{4}\left(v^{2}+\omega^{2}\right) Y^{+} Y^{-}+\frac{g^{2}}{4}\left(u^{2}+\omega^{2}\right) X^{0 *} X^{0}+\frac{1}{2}\left(A_{3} A_{8} B C\right) M^{2}\left(\begin{array}{c}
A_{3} \\
A_{8} \\
B \\
C
\end{array}\right),
$$

where the Lorentz indices have been omitted and should be understood. The squared-mass matrix of the neutral gauge bosons is found to be

$$
M^{2}=\frac{g^{2}}{2}\left(\begin{array}{cccc}
\frac{1}{2}\left(u^{2}+v^{2}\right) & \frac{u^{2}-v^{2}}{2 \sqrt{3}} & -\frac{t_{X}\left(u^{2}+2 v^{2}\right)}{3} & \frac{t_{N}\left(u^{2}-v^{2}\right)}{3} \\
\frac{u^{2}-v^{2}}{2 \sqrt{3}} & \frac{1}{6}\left(u^{2}+v^{2}+4 \omega^{2}\right) & -\frac{t_{X}\left(u^{2}-2\left(v^{2}+\omega^{2}\right)\right)}{3 \sqrt{3}} & \frac{t_{N}\left(u^{2}+v^{2}+4 \omega^{2}\right)}{3 \sqrt{3}} \\
-\frac{t_{X}\left(u^{2}+2 v^{2}\right)}{3} & -\frac{t_{X}\left(u^{2}-2\left(v^{2}+\omega^{2}\right)\right)}{3 \sqrt{3}} & \frac{2}{9} t_{X}^{2}\left(u^{2}+4 v^{2}+\omega^{2}\right) & -\frac{2}{9} t_{X} t_{N}\left(u^{2}-2\left(v^{2}+\omega^{2}\right)\right) \\
\frac{t_{N}\left(u^{2}-v^{2}\right)}{3} & \frac{t_{N}\left(u^{2}+v^{2}+4 \omega^{2}\right)}{3 \sqrt{3}} & -\frac{2}{9} t_{X} t_{N}\left(u^{2}-2\left(v^{2}+\omega^{2}\right)\right) & \frac{2}{9} t_{N}^{2}\left(u^{2}+v^{2}+4\left(\omega^{2}+9 \Lambda^{2}\right)\right)
\end{array}\right) .
$$


The non-Hermitian gauge bosons $W^{ \pm}, X^{0,0 *}$, and $Y^{ \pm}$are physical fields with corresponding masses:

$$
\begin{gathered}
m_{W}^{2}=\frac{g^{2}}{4}\left(u^{2}+v^{2}\right), \quad m_{X}^{2}=\frac{g^{2}}{4}\left(u^{2}+\omega^{2}\right), \\
m_{Y}^{2}=\frac{g^{2}}{4}\left(v^{2}+\omega^{2}\right) .
\end{gathered}
$$

Because of the constraints $u, v \ll \omega$, we have $m_{W} \ll m_{X} \simeq m_{Y}$. The $W$ boson is identified as the SM $W$ boson. It follows

$$
u^{2}+v^{2}=(246 \mathrm{GeV})^{2} .
$$

The $X$ and $Y$ fields are the new gauge bosons with the large masses given in the $\omega$ scale. The physical charged gauge bosons and their masses are summarized in Table III.

It is worth mentioning that after diagonalization, in the obtained masses of gauge bosons, there is no mixing among the VEVs, i.e., in the expression of squared masses, there are no terms such as $u v, u \omega, v \omega$, etc. For more details, the reader is referred to Ref. [9].

From the aforementioned analysis, it follows that the phenomenological aspects of the 3-3-1-1 model can be divided into two pictures corresponding to different domain values of VEVs.

\section{Picture (i): $\Lambda \sim \omega \gg v \sim u$}

The physical neutral gauge bosons are derived through the following transformation $\left(A_{3}, A_{8}, B, C\right) \rightarrow$ $\left(A, Z, Z_{2}, Z_{1}\right)$ :

$$
\left(\begin{array}{c}
A_{3} \\
A_{8} \\
B \\
C
\end{array}\right)=U_{1} U_{2} U_{3}\left(\begin{array}{c}
A \\
Z \\
Z_{2} \\
Z_{1}
\end{array}\right) .
$$

TABLE III. The mass of charged gauge bosons.

\begin{tabular}{lccc}
\hline \hline Gauge boson & $W$ & $Y$ & $X$ \\
\hline Squared mass & $\frac{g^{2}}{4}\left(u^{2}+v^{2}\right)$ & $\frac{g^{2}}{4}\left(\omega^{2}+v^{2}\right)$ & $\frac{g^{2}}{4}\left(\omega^{2}+u^{2}\right)$ \\
\hline \hline
\end{tabular}

The above diagonalization is realized through three steps [28-31],

The first step: $M^{\prime 2}=U_{1}^{T} M^{2} U_{1}$,

The second step: $M^{\prime \prime 2}=U_{2}^{T} M^{\prime 2} U_{2}$,

The final step: $M^{\prime \prime \prime 2}=U_{3}^{T} M^{\prime \prime 2} U_{3}=\operatorname{diag}\left(0, m_{Z}^{2}, m_{Z_{2}}^{2}, m_{Z_{1}}^{2}\right)$,

where

$U_{1}=\left(\begin{array}{cccc}s_{W} & c_{W} & 0 & 0 \\ -\frac{s_{W}}{\sqrt{3}} & \frac{s_{W} t_{W}}{\sqrt{3}} & \sqrt{1-\frac{t_{W}^{2}}{3}} & 0 \\ c_{W} \sqrt{1-\frac{t_{W}^{2}}{3}} & -s_{W} \sqrt{1-\frac{t_{W}^{2}}{3}} & \frac{t_{W}}{\sqrt{3}} & 0 \\ 0 & 0 & 0 & 1\end{array}\right)$,
$U_{2} \simeq\left(\begin{array}{ccc}1 & 0 & 0 \\ 0 & 1 & \mathcal{E} \\ 0 & -\mathcal{E}^{T} & 1\end{array}\right)$,
$U_{3}=\left(\begin{array}{cccc}1 & 0 & 0 & 0 \\ 0 & 1 & 0 & 0 \\ 0 & 0 & c_{\xi} & -s_{\xi} \\ 0 & 0 & s_{\xi} & c_{\xi}\end{array}\right)$.

In Eq. (25), the $\mathcal{E}$ is a two-component vector given by [28-31]

$$
\begin{aligned}
\mathcal{E}_{1} & =-\frac{\sqrt{4 t_{X}^{2}+3}\left\{3 \Lambda^{2}\left[\left(2 t_{X}^{2}-3\right) u^{2}+\left(4 t_{X}^{2}+3\right) v^{2}\right]+t_{X}^{2} \omega^{2}\left(u^{2}+v^{2}\right)\right\}}{4 \Lambda^{2}\left(t_{X}^{2}+3\right)^{2} \omega^{2}} \ll 1, \\
\mathcal{E}_{2} & =\frac{t_{X}^{2} \sqrt{4 t_{X}^{2}+3}\left(u^{2}+v^{2}\right)}{8 \Lambda^{2}\left(t_{X}^{2}+3\right)^{3 / 2} t_{N}} \ll 1, \\
t_{2 \xi} & \simeq \frac{4 \sqrt{3+t_{X}^{2}} t_{N} \omega^{2}}{\left(3+t_{X}^{2}\right) \omega^{2}-4 t_{N}^{2}\left(\omega^{2}+9 \Lambda^{2}\right)}, \\
s_{W} & =\frac{\sqrt{3} t_{X}}{\sqrt{3+4 t_{X}^{2}}} \simeq \sqrt{0.231} .
\end{aligned}
$$

Finally we obtain the masses of neutral gauge bosons as follows

$$
m_{Z}^{2} \simeq \frac{g^{2}\left(u^{2}+v^{2}\right)}{4 c_{W}^{2}},
$$




$$
\begin{aligned}
& m_{Z_{1}}^{2} \simeq \frac{g^{2}}{18}\left(\left(3+t_{X}^{2}\right) \omega^{2}+4 t_{N}^{2}\left(\omega^{2}+9 \Lambda^{2}\right)+\sqrt{\left.\left(\left(3+t_{X}^{2}\right) \omega^{2}-4 t_{N}^{2}\left(\omega^{2}+9 \Lambda^{2}\right)\right)^{2}+16\left(3+t_{X}^{2}\right) t_{N}^{2} \omega^{4}\right)},\right. \\
& m_{Z_{2}}^{2} \simeq \frac{g^{2}}{18}\left(\left(3+t_{X}^{2}\right) \omega^{2}+4 t_{N}^{2}\left(\omega^{2}+9 \Lambda^{2}\right)-\sqrt{\left.\left(\left(3+t_{X}^{2}\right) \omega^{2}-4 t_{N}^{2}\left(\omega^{2}+9 \Lambda^{2}\right)\right)^{2}+16\left(3+t_{X}^{2}\right) t_{N}^{2} \omega^{4}\right)} .\right.
\end{aligned}
$$

From the experimental data $\Delta \rho<0.0007$, ones get $u / \omega<$ 0.0544 or $\omega>3.198 \mathrm{TeV}$ [27] (provided that $u=$ $246 / \sqrt{2} \mathrm{GeV}$ as mentioned). Therefore, the value of $\omega$ results in the $\mathrm{TeV}$ scale as expected.

It has been shown that the ordinary 3-3-1 models are only effective theory, as the B-L charge and the unitarity are violated [34]. For the case $\Lambda \gg w$, the limit of the 3-3-1 breaking scale followed from flavor changing neutral currents as well as LEPII searches is $w>3.6 \mathrm{TeV}$. Due to an extra $U(1)_{N}$ subgroup, the kinetic terms give an effect on the $\rho$ parameter. It is well known that the radiative correction of heavy particles groups into Peskin-Takeuchi $S, T, U$ parameters $[35,36]$. In the frameworks of the 3-3-1 models, the above parameters were investigated in Refs. [30,34,37] It was remarked that if $\Lambda \gg w$, the $\Delta \rho$ relating to the oblique parameter $T$ depends only on $w$ and $\beta$-the parameter appeared in the electric charge operator, not on $\Lambda, \frac{g_{N}}{g}$ and on $\delta$-the coefficient of mixing between $B_{\mu \nu}$ and $C_{\mu \nu}$ [30]. In the case of $\Lambda \gg w$, the result is the same as before, i.e., $w>3.6 \mathrm{TeV}$. In the case $\Lambda=2 w$, the value of $w$ ranges from 3 to $3.5 \mathrm{TeV}$.

From LHC searches, it follows that the lower bound on the $Z^{\prime}$ boson mass in 3-3-1 models is around $2.5 \mathrm{TeV}$ [38]. Hence, the 3-3-1 scale $\omega$ is about $6.3 \mathrm{TeV}$. In addition, from the decays $B_{s, d} \rightarrow \mu^{+} \mu^{-}$and $B_{d} \rightarrow K^{*}(K) \mu^{+} \mu^{-}$[39-43] it follows that the lower limit on the $Z^{\prime}$ boson mass ranges from $1 \mathrm{TeV}$ to $3 \mathrm{TeV}$. Hence, both ordinary 3-3-1 and 3-3-1-1 models provide the similar bound on $\omega$.

\section{Picture (ii): $\Lambda \gg \omega \gg v \sim u$}

If we assume $\Lambda \gg \omega \gg u \sim v$, three gauge bosons are derived as [28-31]

$$
\begin{gathered}
m_{Z}^{2} \simeq \frac{g^{2}\left(u^{2}+v^{2}\right)}{4 c_{W}^{2}}, \\
m_{Z_{1}}^{2} \simeq 4 g^{2} t_{2}^{2} \Lambda^{2}, \\
m_{Z_{2}}^{2} \simeq \frac{g^{2} c_{W}^{2} \omega^{2}}{\left(3-4 s_{W}^{2}\right)} .
\end{gathered}
$$

From the Table III and Eqs. (29)-(31), the $W^{ \pm}$boson and the $Z$ boson are recognized as two famous gauge bosons in the SM. Now we turn to the main object-the effective potential.

\section{EFFECTIVE POTENTIAL}

Within the above assumption, the Higgs potential is given as follows [27-31],

$$
\begin{aligned}
V(\rho, \eta, \chi, \phi)= & \mu_{1}^{2} \rho^{\dagger} \rho+\mu_{2}^{2} \chi^{\dagger} \chi+\mu_{3}^{2} \eta^{\dagger} \eta+\lambda_{1}\left(\rho^{\dagger} \rho\right)^{2}+\lambda_{2}\left(\chi^{\dagger} \chi\right)^{2}+\lambda_{3}\left(\eta^{\dagger} \eta\right)^{2}+\lambda_{4}\left(\rho^{\dagger} \rho\right)\left(\chi^{\dagger} \chi\right)+\lambda_{5}\left(\rho^{\dagger} \rho\right)\left(\eta^{\dagger} \eta\right)+\lambda_{6}\left(\chi^{\dagger} \chi\right)\left(\eta^{\dagger} \eta\right) \\
& +\lambda_{7}\left(\rho^{\dagger} \chi\right)\left(\chi^{\dagger} \rho\right)+\lambda_{8}\left(\rho^{\dagger} \eta\right)\left(\eta^{\dagger} \rho\right)+\lambda_{9}\left(\chi^{\dagger} \eta\right)\left(\eta^{\dagger} \chi\right)+\mu^{2} \phi^{\dagger} \phi+\lambda\left(\phi^{\dagger} \phi\right)^{2}+\lambda_{10}\left(\phi^{\dagger} \phi\right)\left(\rho^{\dagger} \rho\right) \\
& +\lambda_{11}\left(\phi^{\dagger} \phi\right)\left(\chi^{\dagger} \chi\right)+\lambda_{12}\left(\phi^{\dagger} \phi\right)\left(\eta^{\dagger} \eta\right),
\end{aligned}
$$

from which, ones obtain $V_{0}$ depending on VEVs:

$$
\begin{aligned}
V_{0}= & \frac{\lambda \phi_{\Lambda}^{4}}{4}+\frac{1}{4} \lambda_{11} \phi_{\Lambda}^{2} \phi_{\omega}^{2}+\frac{\lambda_{2} \phi_{\omega}^{4}}{4}+\frac{\phi_{\Lambda}^{2} \mu^{2}}{2}+\frac{1}{2} \mu_{2}^{2} \phi_{\omega}^{2}+\frac{\lambda_{3} \phi_{u}^{4}}{4}+\frac{1}{4} \lambda_{12} \phi_{\Lambda}^{2} \phi_{u}^{2}+\frac{1}{4} \lambda_{6} \phi_{u}^{2} \phi_{\omega}^{2} \\
& +\frac{1}{2} \mu_{3}^{2} \phi_{u}^{2}+\frac{1}{4} \lambda_{5} \phi_{u}^{2} \phi_{v}^{2}+\frac{\lambda_{1} \phi_{v}^{4}}{4}+\frac{1}{4} \lambda_{10} \phi_{\Lambda}^{2} \phi_{v}^{2}+\frac{1}{4} \lambda_{4} \phi_{v}^{2} \phi_{\omega}^{2}+\frac{1}{2} \mu_{1}^{2} \phi_{v}^{2} .
\end{aligned}
$$

Here $V_{0}$ has quartic form like in the SM, but it depends on four variables $\phi_{\Lambda}, \phi_{\omega}, \phi_{u}, \phi_{v}$, and has the mixing terms between them. However, developing the potential (32), we obtain four minimum equations. Therefore, we can transform the mixing between four variables to the form depending only on $\phi_{\Lambda}, \phi_{\omega}, \phi_{u}$, and $\phi_{v}$. Let us explain this point in detail. The minimum conditions eliminate the mixing only inside the actual VEV. The above mentioned mixing is due to couplings between fields of the Higgs potential. The Higgs masses do not have the mixing of VEVs when the fields are inside actual VEV. Outside VEV, the fields do not have masses. Hence the symmetry is restored; and consequently the EWPT does not exist.

Furthermore, importantly, there are the mixings of VEVs because of the unwanted terms such as $\lambda_{4}\left(\rho^{\dagger} \rho\right)\left(\chi^{\dagger} \chi\right)$, $\lambda_{5}\left(\rho^{\dagger} \rho\right)\left(\eta^{\dagger} \eta\right), \lambda_{6}\left(\chi^{\dagger} \chi\right)\left(\eta^{\dagger} \eta\right), \lambda_{7}\left(\rho^{\dagger} \chi\right)\left(\chi^{\dagger} \rho\right), \lambda_{8}\left(\rho^{\dagger} \eta\right)\left(\eta^{\dagger} \rho\right)$, 


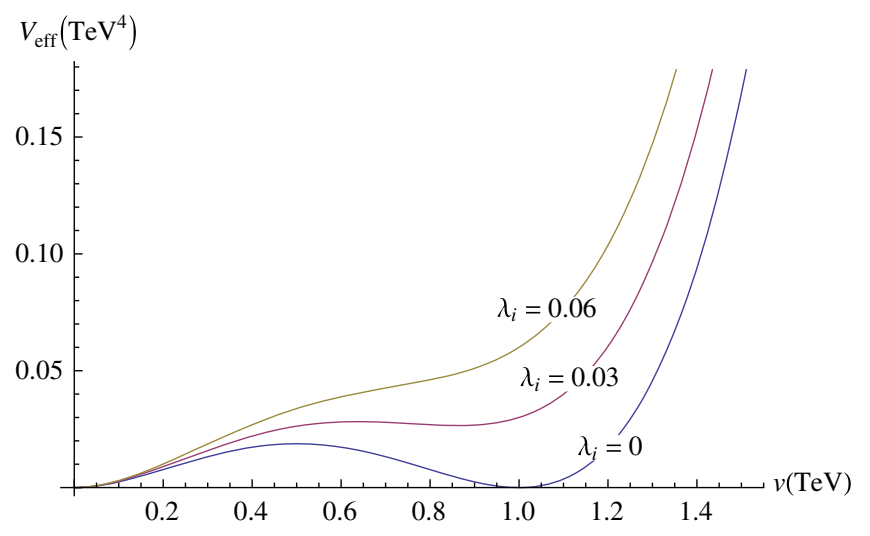

FIG. 1. The contours of the effective potential in (34) as a function of $v$ for some values of $\lambda_{i}$ as $\lambda=0.3, D=0.3, E=$ $0.6, \Lambda^{2}+\omega^{2}+v^{2}=1 \mathrm{TeV}^{2}$.

$\lambda_{9}\left(\chi^{\dagger} \eta\right)\left(\eta^{\dagger} \chi\right), \quad \lambda_{10}\left(\phi^{\dagger} \phi\right)\left(\rho^{\dagger} \rho\right), \quad \lambda_{11}\left(\phi^{\dagger} \phi\right)\left(\chi^{\dagger} \chi\right), \quad$ and $\lambda_{12}\left(\phi^{\dagger} \phi\right)\left(\eta^{\dagger} \eta\right)$ in Eq. (32). To satisfy the generation of inflation with $\phi$-inflaton [28,31], the values $\lambda_{10,11,12}$ can be small, is about $10^{-10}-10^{-6}$. Thus, $\lambda_{4,5,6,7,8,9}$ must be also small to make the corrections of high order interactions of the Higgs will not be divergent.

In general, if we did not neglect these mixings, $V_{0}$ will have additional components $\Lambda v, \Lambda \omega, \omega v, u v$. At the temperature $\mathrm{T}$, for instance, the effective potential depending on VEV $v$, will be an example form:

$$
\begin{aligned}
V_{\mathrm{eff}}(v) & =\lambda v^{4}-E v^{3}+D v^{2}+\lambda_{k} \cdot \omega^{2} v^{2}+\lambda_{j} \cdot \Lambda^{2} v^{2}+u^{2} \cdot v^{2} \\
& \approx \lambda v^{4}-E v^{3}+D v^{2}+\lambda_{i} \cdot\left(\omega^{2}+\Lambda^{2}+u^{2}\right) v^{2}
\end{aligned}
$$

The contours of the effective potential in (34) at $\omega^{2}+\Lambda^{2}+$ $u^{2}=1 \mathrm{TeV}^{2}$ as a function of $v$ for some values of $\lambda_{i}$ is plotted in Fig. 1.

From Fig. 1, we see that at arbitrary temperature $T$ when $\lambda_{i}, i=4, . ., 9$ increases, the second minimum of the effective potential fades. For a first order phase transition, the value of $\lambda_{i}$ is not too large, so that the potential still has two minima. We observe that if $\lambda_{i}$ is enough small to have a second minimum, at arbitrary temperature, the shape of the effective potential remains the same in the absence of $\lambda_{i}$. Therefore, we have one more reason to assume that $\lambda_{i}$ must be small and this mixing can be neglected. Hence, we can write $V_{0}\left(\phi_{\Lambda}, \phi_{\omega}, \phi_{u}, \phi_{v}\right)=V_{0}\left(\phi_{\Lambda}\right)+V_{0}\left(\phi_{\omega}\right)+V_{0}\left(\phi_{u}\right)+$ $V_{0}\left(\phi_{v}\right)$ and ignore the mixing of different VEVs, otherwise our phase transitions will be very complex or distorted.

In order to derive effective potential, we need the mass spectrum of fields. Starting from the Lagrangian of the scalars (both kinetic and potential terms) and Yukawa interactions, and expanding Higgs fields around VEVs, we obtain the mass terms for all fields in the 3-3-1-1 model.

The gauge sector in the 3-3-1-1 has ten gauge bosons: the photon and nine massive gauge bosons. The latter includes two massive like the SM $Z$ and $W^{ \pm}$bosons, and two new heavy neutral $Z_{1}, Z_{2}$ bosons, the charged gauge bosons $Y^{ \pm}$ and the neutral non-Hermitian bosons: $X^{0,0^{*}}$. The Higgs sector contains four charged Higgs bosons $H_{1}^{ \pm}, H_{2}^{ \pm}$, seven neutral Higgs bosons $S_{4}, A_{\eta}^{\prime}, A_{\chi}, S_{\eta}, S_{\chi}^{\prime}, S_{\rho}, H_{3}$. The model consists of four heavy quarks $U, D_{1}, D_{2}$, top quark. Masses of fields in the 3-3-1-1 model are presented in Table IV.

From the mass spectra, we can split masses of particles into four parts as follows

$$
\begin{aligned}
m^{2}\left(\phi_{\Lambda}, \phi_{\omega}, \phi_{u}, \phi_{v}\right)= & m^{2}\left(\phi_{\Lambda}\right)+m^{2}\left(\phi_{\omega}\right)+m^{2}\left(\phi_{u}\right) \\
& +m^{2}\left(\phi_{v}\right) .
\end{aligned}
$$

Taking into account Eqs. (33) and (35), we can also split the effective potential into four parts

$$
\begin{aligned}
V_{\text {eff }}\left(\phi_{\Lambda}, \phi_{\omega}, \phi_{u}, \phi_{v}\right)= & V_{\text {eff }}\left(\phi_{\Lambda}\right)+V_{\text {eff }}\left(\phi_{\omega}\right)+V_{\text {eff }}\left(\phi_{u}\right) \\
& +V_{\text {eff }}\left(\phi_{v}\right)
\end{aligned}
$$

It is difficult to study the electroweak phase transition with four VEVs, so we assume $\phi_{\Lambda} \approx \phi_{\omega}, \phi_{u} \approx \phi_{v}$ over spacetimes. Then, the effective potential becomes

TABLE IV. Mass formulas of particles in 3-3-1-1 model.

\begin{tabular}{lcccccc}
\hline \hline Boson & $W^{ \pm}$ & $Y^{ \pm}$ & $X$ & $Z$ & $Z_{1}$ & $Z_{2}$ \\
\hline Squared mass & $\frac{g^{2}}{4}\left(\phi_{u}^{2}+\phi_{v}^{2}\right)$ & $\frac{g^{2}}{4}\left(\phi_{\omega}^{2}+\phi_{v}^{2}\right)$ & $\frac{g^{2}}{4}\left(\phi_{\omega}^{2}+\phi_{u}^{2}\right)$ & & & \\
Picture (i) & & & Eq. (26) & Eq. (27) & Eq. (28) \\
Picture (ii) & & & $\frac{g^{2}\left(\phi_{u}^{2}+\phi_{v}^{2}\right)}{4 c_{\omega}^{2}}$ & $4 g^{2} t_{N}^{2} \phi_{\Lambda}^{2}$ & $\frac{g^{2} c_{W}^{2} \phi_{\omega}^{2}}{3-4 s_{W}^{2}}$ \\
Neutral Higgs boson & $S_{\chi}^{\prime}$ & $S_{\rho}$ & $S_{4}$ & $A_{\eta}^{\prime}$ & $A_{\chi}$ & $S_{\eta}$ \\
Squared mass & $2 \lambda_{2} \phi_{\omega}^{2}$ & $2 \lambda_{1} \phi_{v}^{2}$ & $2 \lambda \phi_{\Lambda}^{2}$ & $\frac{\lambda_{g} \phi_{\omega}^{2}}{2}$ & $\frac{\lambda_{g} \phi_{u}^{2}}{2}$ & $2 \lambda_{3} \phi_{u}^{2}$ \\
Charged Higgs boson & $H_{1}$ & $H_{2}$ & $H_{3}$ & & \\
Complex Higgs boson & $\frac{\phi_{u}^{2}+\phi_{v}^{2}}{2} \lambda_{8}$ & $\frac{\phi_{\omega}^{2}+\phi_{v}^{2}}{2} \lambda_{7}$ & $\frac{\lambda_{g}\left(\phi_{u}^{2}+\phi_{\omega}^{2}\right)}{2}$ & & \\
Squared mass & $\mathrm{U}$ & $D_{1}$ & $D_{2}$ & & \\
Quark & $\frac{1}{2} h^{U 2} \phi_{\omega}^{2}$ & $\frac{1}{2} h_{11}^{D^{2}} \phi_{\omega}^{2}$ & $\frac{1}{2} h_{22}^{D^{2}} \phi_{\omega}^{2}$ & $\frac{1}{2} h_{t}^{2} \phi_{u}^{2}$ & \\
Squared mass & \multicolumn{5}{c}{} \\
\hline \hline
\end{tabular}




$$
V_{\mathrm{eff}}\left(\phi_{\Lambda}, \phi_{\omega}, \phi_{u}, \phi_{v}\right)=V_{\mathrm{eff}}\left(\phi_{\omega}\right)+V_{\mathrm{eff}}\left(\phi_{u}\right) .
$$

From Table IV, it follows that the squared masses of gauge and Higgs bosons are split into three separated parts corresponding to three SSB stages. It is consistent with the analysis given in Ref. [9].

It is interesting to note that the way of splitting into two or three phases performed in this paper is similar to that in Ref. [44]. However, in this paper, the multiperiodicity of phase transition is shown more transparently both in its title and as well as in content.

\section{ELECTROWEAK PHASE TRANSITION WITHOUT NEUTRAL FERMION}

Taking phase transitions in this model into account, it is important to find the activity domain of $\omega, \Lambda, u$ and $v$. Looking at data in Ref. [45,46], we arrive to assumption: $m_{Z_{2}} \geq 2.2 \mathrm{TeV}$. In addition, from Ref. [27], we also assume $m_{Z_{2}}<2.5 \mathrm{TeV}$. Hence

$$
2.2 \mathrm{TeV} \leq m_{Z_{2}} \leq 2.5 \mathrm{TeV} .
$$

From the constraint in (36), we will infer the domain values of $\omega$ and $\Lambda$. It is worth mentioning that in the 3-3-1-1 model, the structure of symmetry breaking which can be divided into two or three periods depending on scale of VEVs as suggesting in the above two pictures.

\section{A. Two periods EWPT in picture (i)}

In picture (i), we have assumed $\Lambda \sim \omega \gg u \sim v$ meaning that the symmetry breaking or phase transition has two periods. The first transition is $S U(3) \rightarrow S U(2)$ through $\omega \sim \Lambda$, which generates masses of the heavy gauge bosons $X^{ \pm}, Y^{ \pm}, Z_{1}, Z_{2}$, Higgs bosons $H_{2}, H_{3}, A_{\eta}^{\prime}, S^{\prime} \chi, S_{4}$, and three exotic quarks. The phase transition $S U(3) \rightarrow S U(2)$ only depends on $\phi_{\omega} \sim \phi_{\Lambda}$.

When our universe has been expanding and cooling due to $u$ scale, the symmetry breaking $S U(2) \rightarrow U(1)$ is turned on, which generates masses of the SM particles and the last part of masses of $\mathrm{H}_{2}, \mathrm{H}_{3}, X^{ \pm}, Y^{ \pm}$. Therefore, phase transition $S U(2) \rightarrow U(1)$ only depends on $\phi_{u} \sim \phi_{v}$.

\section{Phase transition $S U(3) \rightarrow S U(2)$}

This phase transition involves exotic quarks, heavy bosons, but excludes the SM particles. As a consequence, the effective potential of the EWPT $S U(3) \rightarrow U(1)$ is $V_{\text {eff }}\left(\phi_{\omega}\right)$.

Applying the Coleman-Weinberg's method, the effective potential $V_{\text {eff }}\left(\phi_{\omega}\right)$ is given as

$$
V_{\mathrm{eff}}\left(\phi_{\omega}\right)=D_{\omega}\left(T^{2}-T_{0 \omega}^{2}\right) \phi_{\omega}^{2}-E_{\omega} T \phi_{\omega}^{3}+\frac{\lambda_{\omega}(T)}{4} \phi_{\omega}^{4},
$$

where

$$
\begin{aligned}
& \lambda_{\omega}(T)=-\frac{m_{A_{\eta}^{\prime}}^{4} \log \left(\frac{m_{A_{\eta}^{\prime}}^{2}}{T^{2} a_{b}}\right)}{16 \pi^{2} \omega^{4}}-\frac{m_{H_{2}}^{4} \log \left(\frac{m_{H_{2}}^{2}}{T^{2} a_{b}}\right)}{8 \pi^{2} \omega^{4}}-\frac{m_{H_{3}}^{4} \log \left(\frac{m_{H_{3}}^{2}}{T^{2} a_{b}}\right)}{16 \pi^{2} \omega^{4}}-\frac{m_{S_{\chi}^{\prime}}^{4} \log \left(\frac{m_{S_{x}^{\prime}}^{2}}{T^{2} a_{b}}\right)}{16 \pi^{2} \omega^{4}}-\frac{m_{S_{4}}^{4} \log \left(\frac{m_{S_{4}}^{2}}{T^{2} a_{b}}\right)}{16 \pi^{2} \omega^{4}}-\frac{3 m_{X}^{4} \log \left(\frac{m_{X}^{2}}{T^{2} a_{b}}\right)}{8 \pi^{2} \omega^{4}} \\
& -\frac{3 m_{Y}^{4} \log \left(\frac{m_{Y}^{2}}{T^{2} a_{b}}\right)}{8 \pi^{2} \omega^{4}}-\frac{3 m_{Z_{1}}^{4} \log \left(\frac{m_{Z_{1}}^{2}}{T^{2} a_{b}}\right)}{16 \pi^{2} \omega^{4}}-\frac{3 m_{Z_{2}}^{4} \log \left(\frac{m_{Z_{2}}^{2}}{T^{2} a_{b}}\right)}{16 \pi^{2} \omega^{4}}+\frac{3 M_{D_{1}}^{4} \log \left(\frac{M_{D_{1}}^{2}}{T^{2} a_{f}}\right)}{4 \pi^{2} \omega^{4}}+\frac{3 M_{D_{2}}^{4} \log \left(\frac{M_{D_{2}}^{2}}{T^{2} a_{f}}\right)}{4 \pi^{2} \omega^{4}} \\
& +\frac{3 M_{U}^{4} \log \left(\frac{M_{U}^{2}}{T^{2} a_{f}}\right)}{4 \pi^{2} \omega^{4}}+\frac{m_{A_{\eta}^{\prime}}^{2}}{2 \omega^{2}}+\frac{m_{H_{3}}^{2}}{2 \omega^{2}}+\frac{m_{S_{x}^{\prime}}^{2}}{2 \omega^{2}}+\frac{m_{S_{4}}^{2}}{2 \omega^{2}}, \\
& E_{\omega}=\frac{m_{A_{\eta}^{\prime}}^{3}}{12 \pi \omega^{3}}+\frac{m_{H_{2}}^{3}}{6 \pi \omega^{3}}+\frac{m_{H_{3}}^{3}}{12 \pi \omega^{3}}+\frac{m_{S_{\chi}^{\prime}}^{3}}{12 \pi \omega^{3}}+\frac{m_{S_{4}}^{3}}{12 \pi \omega^{3}}+\frac{m_{X}^{3}}{2 \pi \omega^{3}}+\frac{m_{Y}^{3}}{2 \pi \omega^{3}} \\
& +\frac{m_{Z_{1}^{3}}}{4 \pi \omega^{3}}+\frac{m_{Z_{2}}^{3}}{4 \pi \omega^{3}} \\
& D_{\omega}=\frac{m_{A_{\eta}^{\prime}}^{2}}{24 \omega^{2}}+\frac{M_{D_{1}}^{2}}{4 \omega^{2}}+\frac{M_{D_{2}}^{2}}{4 \omega^{2}}+\frac{m_{H_{2}}^{2}}{12 \omega^{2}}+\frac{m_{H_{3}}^{2}}{24 \omega^{2}}+\frac{m_{S_{\chi}^{\prime}}^{2}}{24 \omega^{2}}+\frac{m_{S_{4}}^{2}}{24 \omega^{2}}+\frac{m_{X}^{2}}{4 \omega^{2}}+\frac{m_{Y}^{2}}{4 \omega^{2}}+\frac{m_{Z_{1}}^{2}}{8 \omega^{2}}+\frac{m_{Z_{2}}^{2}}{8 \omega^{2}}+\frac{M_{U}^{2}}{4 \omega^{2}}, \\
& F_{\omega}=\frac{m_{A_{\eta}^{\prime}}^{4}}{32 \pi^{2} \omega^{2}}-\frac{m_{A_{\eta}^{\prime}}^{2}}{4}-\frac{3 M_{D_{1}}^{4}}{8 \pi^{2} \omega^{2}}-\frac{3 M_{D_{2}}^{4}}{8 \pi^{2} \omega^{2}}+\frac{m_{H_{2}}^{4}}{16 \pi^{2} \omega^{2}}+\frac{m_{H_{3}}^{4}}{32 \pi^{2} \omega^{2}}-\frac{m_{H_{3}}^{2}}{4}+\frac{m_{S_{\chi}^{\prime}}^{4}}{32 \pi^{2} \omega^{2}}-\frac{m_{S_{\chi}^{\prime}}^{2}}{4} \\
& +\frac{m_{S_{4}}^{4}}{32 \pi^{2} \omega^{2}}-\frac{m_{S_{4}}^{2}}{4}+\frac{3 m_{X}^{4}}{16 \pi^{2} \omega^{2}}+\frac{3 m_{Y}^{4}}{16 \pi^{2} \omega^{2}}+\frac{3 m_{Z_{1}}^{4}}{32 \pi^{2} \omega^{2}}+\frac{3 m_{Z_{2}}^{4}}{32 \pi^{2} \omega^{2}}-\frac{3 M_{U}^{4}}{8 \pi^{2} \omega^{2}},
\end{aligned}
$$


and

$$
T_{0 \omega}^{2} \equiv-\frac{F_{\omega}}{D_{\omega}}
$$

The minimum conditions are

$$
\begin{aligned}
V_{\mathrm{eff}}(0)=\left.\frac{\partial V_{\mathrm{eff}}\left(\phi_{\omega}\right)}{\partial \phi_{\omega}}\right|_{\omega} & =0 \\
\left.\frac{\partial^{2} V_{\mathrm{eff}}\left(\phi_{\omega}\right)}{\partial \phi_{\omega}^{2}}\right|_{\omega} & =m_{A_{\eta}^{\prime}}^{2}+m_{H_{3}}^{2}+m_{S_{\chi}^{\prime}}^{2}+m_{S_{4}}^{2} .
\end{aligned}
$$

The values of $V_{\text {eff }}\left(\phi_{\omega}\right)$ at the two minima become equal at the critical temperature and the phase transition strength are

$$
\begin{aligned}
T_{c \omega} & =\frac{T_{0 \omega}}{\sqrt{1-E_{\omega}^{2} / D_{\omega} \lambda_{T_{c \omega}}}}, \\
S_{\omega} & =\frac{2 E_{\omega}}{\lambda_{T_{c \omega}}}
\end{aligned}
$$

From Eqs. (26)-(28), with the limit of $m_{Z_{2}}$ given in Eq. (36), it follows: $5.856 \mathrm{TeV} \leq \omega \sim \Lambda \leq 6.654 \mathrm{TeV}$.

In this work, we assume $\omega=6 \mathrm{TeV}$, so that $m_{Z_{1}}=$ $8.304 \mathrm{TeV}$ and $m_{Z_{2}}=2.254 \mathrm{TeV}$. The problem here is that there are nine variables: the masses of $U, D_{1}, D_{2}, H_{2}, H_{3}$ and $A_{\eta}^{\prime}, S_{\chi}^{\prime}, S_{4}, Z_{1}$. However, for simplicity, we assume $m_{U}=m_{D_{1}}=m_{D_{2}}=m_{H_{2}} \equiv O, m_{A_{\eta}^{\prime}}=m_{S_{\gamma}^{\prime}}=m_{H_{3}}=m_{S_{4}} \equiv P$. Consequently, the critical temperature and the phase transition strength are the function of $O$ and $P$; therefore we can rewrite the phase transition strength as follows

$$
S_{\omega}=\frac{2 E_{\omega}}{\lambda_{T_{c \omega}}} \equiv S_{\omega}\left(O, P, S_{\omega}\right) .
$$

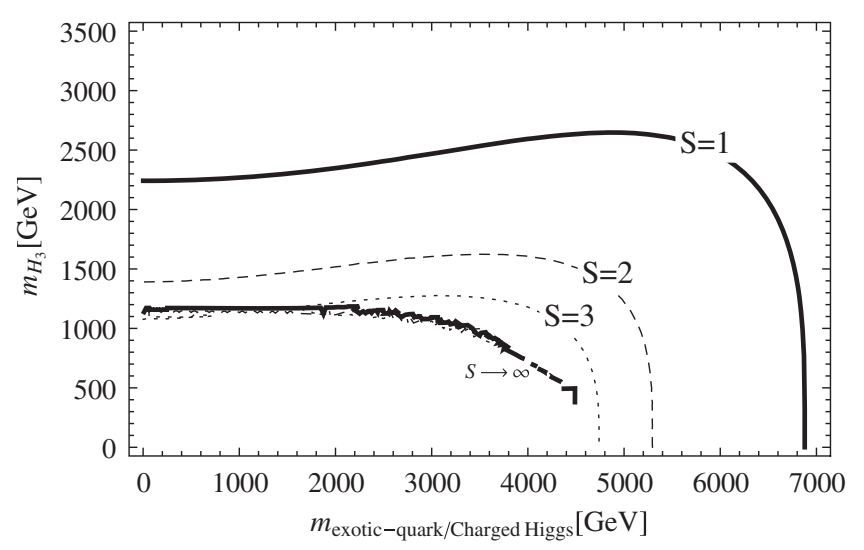

FIG. 2. The mass area corresponds to $S_{\omega}>1$.
In Figs. 2 and 3, we have plotted the relation between masses of the charged particles $O$ and neutral particles $P$ with some values of the phase transition strength at $\omega=6 \mathrm{TeV}$.

The mass region of particles is the largest at $S_{\omega}=1$, the mass region of charged particles and neutral particles are

$$
\begin{aligned}
& 0 \leq m_{\text {Exotic Quark/Charged Higgsboson }} \leq 7000 \mathrm{GeV}, \\
& 0 \leq m_{H_{3}} \leq 2600 \mathrm{GeV}
\end{aligned}
$$

From Eq. (45) it follows that the maximum of $S_{\omega}$ is around 70 .

\section{Phase transition $S U(2) \rightarrow U(1)$}

In this period, the symmetry breaking scale equals to $u=246 / \sqrt{2}$ and the masses of the SM particles and apart of masses of $X, Y, H_{1}, H_{2}, H_{3}, A_{\chi}, S_{\eta}$ are generated.

There are six variables corresponding to the masses of bosons $H_{1}, H_{2}, A_{\chi}, A_{\eta}, H_{3}, S_{\rho}$. For simplicity, we assume: $m_{H_{1}}=m_{H_{2}} \equiv K, \quad m_{A_{\chi}}=m_{S_{\eta}}=m_{H_{3}} \equiv L$, and $m_{S_{\rho}}=125 \mathrm{GeV}$.

The effective potential of EWPT $S U(2) \rightarrow U(1)$ is given as

$$
V_{\mathrm{eff}}\left(\phi_{u}\right)=\frac{\lambda_{u}(T)}{4} \phi_{u}^{4}-E_{u} T \phi_{u}^{3}+D_{u} T^{2} \phi_{u}^{2}+F_{u} \phi_{u}^{2} .
$$

The minimum conditions are

$$
\begin{gathered}
V_{\mathrm{eff}}(0)=\left.\frac{\partial V_{\mathrm{eff}}\left(\phi_{u}\right)}{\partial \phi_{u}}\right|_{u}=0 \\
\left.\frac{\partial^{2} V_{\mathrm{eff}}\left(\phi_{u}\right)}{\partial \phi_{u}^{2}}\right|_{u}=m_{A_{\chi}}^{2}+m_{H_{3}}^{2}+m_{S_{\eta}}^{2}+m_{S_{\rho}}^{2},
\end{gathered}
$$

where

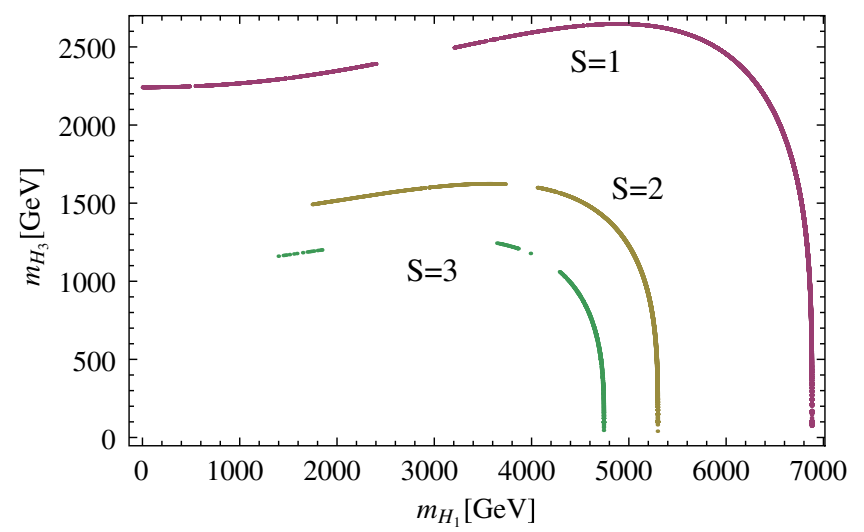

FIG. 3. The mass area corresponds to $S_{\omega}>1$ with real $T_{C}$ condition. The gaps on the lines $(S=1,2,3)$ correspond to values making $T_{C}$ to be complex. 


$$
\begin{aligned}
D_{u}= & \frac{m_{A_{\chi}}^{2}}{24 u^{2}}+\frac{m_{H_{1}}^{2}}{12 u^{2}}+\frac{m_{H_{2}}^{2}}{12 u^{2}}+\frac{m_{H_{3}}^{2}}{24 u^{2}}+\frac{m_{S_{\eta}}^{2}}{24 u^{2}}+\frac{m_{S_{\rho}}^{2}}{24 u^{2}}+\frac{m_{W}^{2}}{4 u^{2}}+\frac{m_{X}^{2}}{4 u^{2}}+\frac{m_{Y}^{2}}{4 u^{2}}+\frac{m_{Z}^{2}}{8 u^{2}}+\frac{M_{t}^{2}}{4 u^{2}}, \\
F_{u}= & \frac{m_{A_{\chi}}^{4}}{32 \pi^{2} u^{2}}-\frac{m_{A_{\chi}}^{2}}{4}+\frac{m_{H_{1}}^{4}}{16 \pi^{2} u^{2}}+\frac{m_{H_{2}}^{4}}{16 \pi^{2} u^{2}}+\frac{m_{H_{3}}^{4}}{32 \pi^{2} u^{2}}-\frac{m_{H_{3}}^{2}}{4}-\frac{m_{S_{\eta}}^{2}}{4}-\frac{m_{S_{\rho}}^{2}}{4} \\
& +\frac{m_{S_{\eta}}^{4}}{32 \pi^{2} u^{2}}+\frac{m_{S_{\rho}}^{4}}{32 \pi^{2} u^{2}}+\frac{3 m_{W}^{4}}{16 \pi^{2} u^{2}}+\frac{3 m_{X}^{4}}{16 \pi^{2} u^{2}}+\frac{3 m_{Y}^{4}}{16 \pi^{2} u^{2}}+\frac{3 m_{Z}^{4}}{32 \pi^{2} u^{2}}-\frac{3 M_{t}^{4}}{8 \pi^{2} u^{2}} \\
E_{u}= & \frac{m_{A_{\chi}}^{3}}{12 \pi u^{3}}+\frac{m_{H_{1}}^{3}}{6 \pi u^{3}}+\frac{m_{H_{2}}^{3}}{6 \pi u^{3}}+\frac{m_{H_{3}}^{3}}{12 \pi u^{3}}+\frac{m_{S_{\eta}}^{3}}{12 \pi u^{3}}+\frac{m_{S_{\rho}}^{3}}{12 \pi u^{3}}+\frac{m_{W}^{3}}{2 \pi u^{3}}+\frac{m_{X}^{3}}{2 \pi u^{3}}+\frac{m_{Y}^{3}}{2 \pi u^{3}}+\frac{m_{Z}^{3}}{4 \pi u^{3}}, \\
\lambda_{u}(T)= & -\frac{m_{A_{\chi}}^{4} \log \left(\frac{m_{A_{X}}^{2}}{T^{2} a_{b}}\right)}{16 \pi^{2} u^{4}}-\frac{m_{H_{1}}^{4} \log \left(\frac{m_{H_{1}}^{2}}{T^{2} a_{b}}\right)}{8 \pi^{2} u^{4}}-\frac{m_{H_{2}}^{4} \log \left(\frac{m_{H_{2}}^{2}}{T^{2} a_{b}}\right)}{8 \pi^{2} u^{4}}-\frac{m_{H_{3}}^{4} \log \left(\frac{m_{H_{3}}^{2}}{T^{2} a_{b}}\right)}{16 \pi^{2} u^{4}}-\frac{m_{S_{\eta}}^{4} \log \left(\frac{m_{S_{\eta}}^{2}}{T^{2} a_{b}}\right)}{16 \pi^{2} u^{4}}-\frac{m_{S_{\rho}}^{4} \log \left(\frac{m_{S_{\rho}}^{2}}{T^{2} a_{b}}\right)}{16 \pi^{2} u^{4}} \\
& -\frac{3 m_{W}^{4} \log \left(\frac{m_{W}^{2}}{T^{2} a_{b}}\right)}{8 \pi^{2} u^{4}}-\frac{3 m_{X}^{4} \log \left(\frac{m_{X}^{2}}{T^{2} a_{b}}\right)}{8 \pi^{2} u^{4}}-\frac{3 m_{Y}^{4} \log \left(\frac{m_{Y}^{2}}{T^{2} a_{b}}\right)}{8 \pi^{2} u^{4}}-\frac{3 m_{Z}^{4} \log \left(\frac{m_{Z}^{2}}{T^{2} a_{b}}\right)}{16 \pi^{2} u^{4}}+\frac{3 M_{t}^{4} \log \left(\frac{M_{t}^{2}}{T^{2} a_{f}}\right)}{4 \pi^{2} u^{4}}+\frac{m_{A_{\chi}}^{2}}{2 u^{2}}+\frac{m_{H_{3}}^{2}}{2 u^{2}}+\frac{m_{S_{\eta}}^{2}}{2 u^{2}}+\frac{m_{S_{\rho}}^{2}}{2 u^{2}}
\end{aligned}
$$

The critical temperature and the phase transition strength are given by

$$
T_{c}=\frac{T_{0}}{\sqrt{1-\frac{E^{2}}{D \lambda_{T_{c}}}}}, \quad S=\frac{2 E}{\lambda_{T_{c}}} .
$$

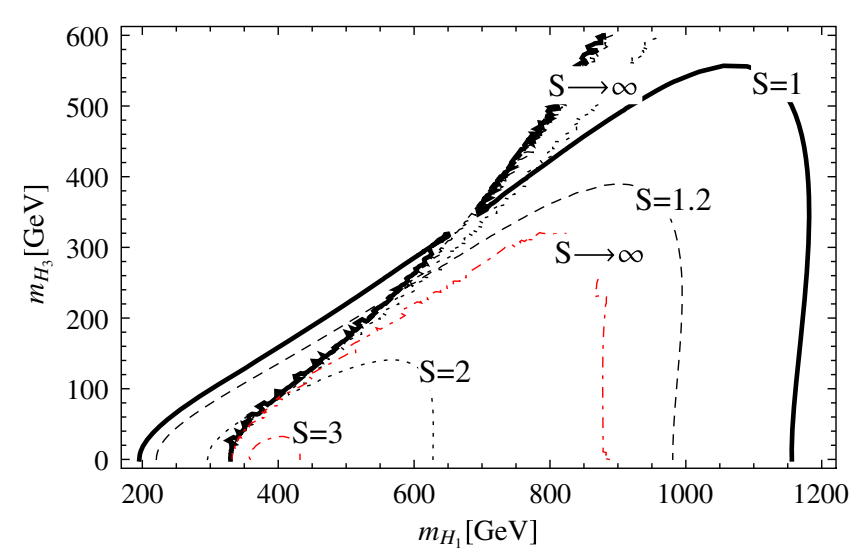

FIG. 4. The strength $S=\frac{2 E_{u}}{\lambda_{T_{c}}}$.

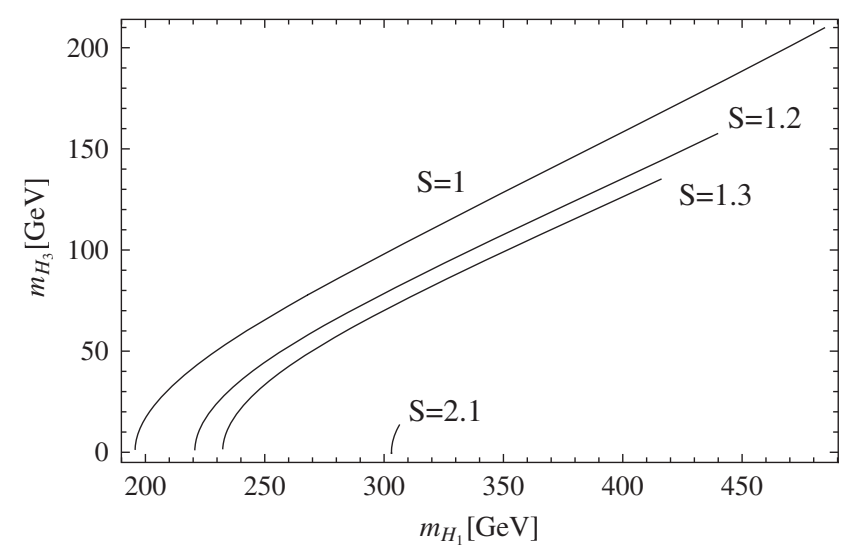

FIG. 5. The strength EWPT $S=\frac{2 E_{u}}{\lambda_{T_{c}}}$ with $T_{c}$ must be real.
Like the phase transition $S U(3) \rightarrow S U(2)$, in Fig. 5 we have plotted the relation between masses of the charged particles $K$ and neutral particles $L$ with some values of the phase transition strength.

However, we can fit the mass of heavy particle one again when considering the condition of $T_{c}$ to be real, so that Fig. 4 is redrew to Fig. 5 and the maximum of strength is reduced from 3 to 2.12 .

The mass region of neutral and charged particles given in Table $\mathrm{V}$ leads the maximum phase transition strength which must be 2.12. This is larger than 1 but the EWPT is not strong.

\section{B. Three period EWPT in picture (ii)}

In picture (ii), $m_{Z_{2}}^{2} \simeq \frac{g^{2} c_{W}^{2} \omega^{2}}{\left(3-4 s_{W}^{2}\right)}$ with the limit of $m_{Z_{2}}$ given in Eq. (36), we obtain $5.53 \mathrm{TeV} \leq \omega \leq 6.3 \mathrm{TeV}$. Therefore, we also assume $\omega=6 \mathrm{TeV}$ in this picture.

Because $\Lambda \gg \omega=6 \mathrm{TeV}$ and $\omega \gg u \sim v$, therefore there are three periods. The first process is $S U(3)_{L} \otimes$ $U(1)_{X} \otimes U(1)_{N} \rightarrow S U(3)_{L} \otimes U(1)_{X}$. The second one is $S U(3)_{L} \otimes U(1)_{X} \rightarrow S U(2)_{L} \otimes U(1)_{X}$. The third process is $S U(2)_{L} \rightarrow U(1)_{Q}$. The third process is like $S U(2) \rightarrow$ $U(1)$ in the picture (i).

The first process is a transition of the symmetry breaking of $U(1)_{N}$ group. It generates mass for $Z_{1}$ through $\Lambda$ or Higgs boson $S_{4}$. The third process is like the $S U(2) \rightarrow$ $U(1)$ EWPT in picture (i). The second process is like the $S U(3) \rightarrow S U(2)$ in picture (i) but it does not involve $Z_{1}$ and $S_{4}$.

TABLE V. Mass limits of particles with $T_{C}>0$.

\begin{tabular}{lcc}
\hline \hline Strength $S$ & $K[\mathrm{GeV}]$ & $L[\mathrm{GeV}]$ \\
\hline $1.0-2.12$ & $195 \leq K \leq 484.5$ & $0 \leq L \leq 209.8$ \\
\hline \hline
\end{tabular}




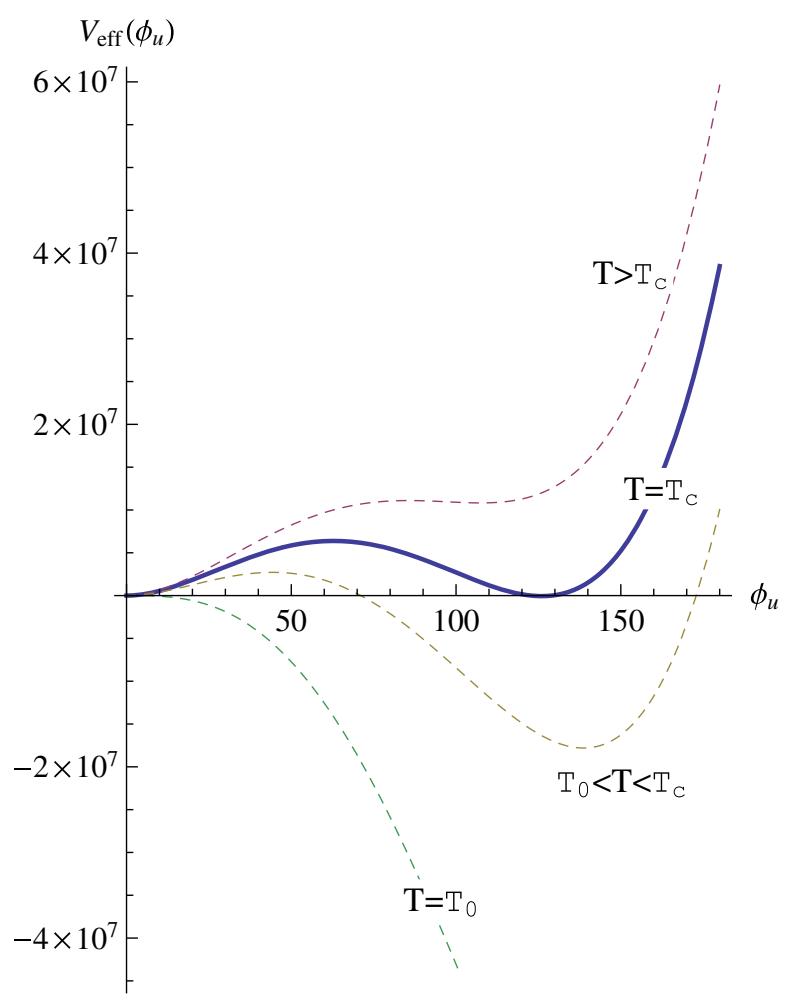

FIG. 6. The strength EWPT $S_{\omega}=\frac{2 E_{\omega}}{\lambda_{T_{c}}}$ with $\omega=6 \mathrm{TeV}$.

The second process has the effective potential is like Eq. (37). In addition, parameters and the minimum conditions are like Eqs. (38), (40)-(44) without $Z_{1}$ and $S_{4}$.

In our numbering process, when we import real $T_{C}$, the mass region of charged and neutral particles are

$$
\begin{aligned}
& 0 \leq m_{\text {Exoticquark/Charged Higgsboson }} \leq 4000 \mathrm{GeV}, \\
& 0 \leq m_{H_{3}} \leq 1000 \mathrm{GeV} .
\end{aligned}
$$

The mass region of charged bosons is narrower than that in the Fig. 6. From Eq. (45), the maximum of $S$ has been estimated to be around 100 .

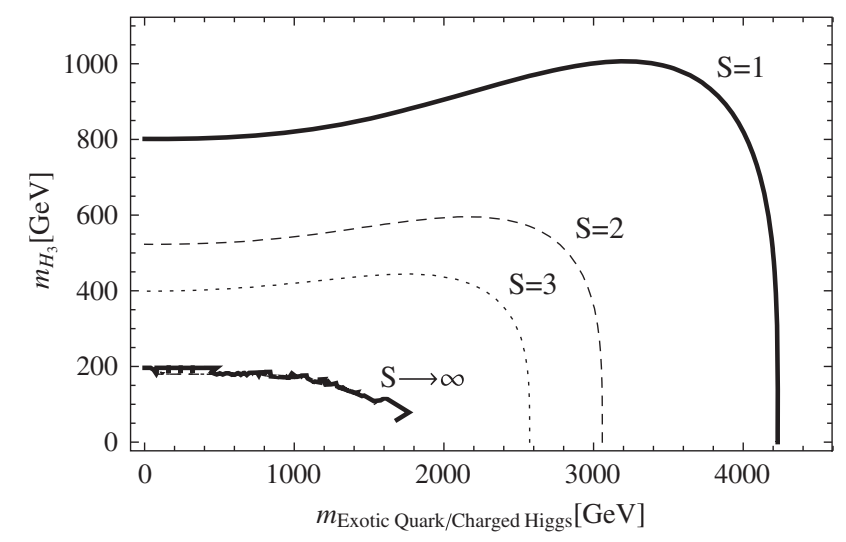

FIG. 7. The strength EWPT $S_{\omega}=\frac{2 E_{u}}{\lambda_{T_{c}}}$ with $\omega=6 \mathrm{TeV}$.

\section{THE ROLE OF NEUTRAL FERMIONS IN EWPT}

The masses of $N_{R}$ can be generated by the scalar content by itself via an effective operator invariant under the 3-3-1-1 symmetry and $W$-parity [27]:

$$
\frac{\lambda_{a b}}{M} \bar{\psi}_{a L}^{C} \psi_{b L}\left(\chi^{\dagger} \chi\right)
$$

The mass scale of $N_{R}$ is unknown, but it can be taken in $\mathrm{TeV}$ scale. However, the analysis of the scattering of $N_{R}$ with distributions of $X, Y, Z_{2}$ bosons given in [27] leads to a consequence that the mass of $N_{R}$ is equal or less than that of the $Z_{2}$ boson as follows

$$
m_{N_{R}}=\frac{m_{Z_{2}}^{2}}{2.557 \mathrm{TeV}} \leq m_{Z_{2}} .
$$

In the $S U(3) \rightarrow S U(2)$, if we add the contribution of neutral fermions, then the maximum of $S$ would decrease. However, the neutral fermions do not lose the first-order EWPT as shown in Table VI.

In Table VI, we have only estimated the maximum strengths and showed that these maximum values are significantly reduced. However, it is very difficult to calculate these values accurately because of the existence of many parameters (the masses of heavy particles); and these values can change slightly (but not too much) with different approximations. Looking at the Table VI, the following remarks are in order:

(1) In case of the neutral fermion absence. In the picture (i), if $Z_{1}$ boson is involved in the $S U(3) \rightarrow S U(2)$ EWPT; the contribution of $Z_{1}$ makes increasing $E$ and $\lambda$, but $\lambda$ increases stronger than $E$. The strength $S=\frac{2 E}{\lambda_{T_{C}}}$ gets the value equals 70 . For the picture (ii), the mentioned value equals 100 .

(2) In case of the neutral fermion existence. When the neutral fermions are involved in both pictures, $S_{\max }$ in picture (ii) decreases faster than $S_{\max }$ in picture (i). The strength gets values equal to 50 and 30 for the picture (i) and (ii), respectively.

If the neutral fermions follow the Fermi-Dirac distribution (i.e., they act as a real fermion but without lepton number), they increase the value of the $\lambda$ and $D$ parameters. Thus, they reduce the value of strength EWPT $S$, because $S=\frac{E}{2 \lambda_{T_{c}}}$ and $E$ do not depend on the neutral fermions.

This suggests that DM candidates are neutral fermions (or fermions in general) which reduce the maximum value of the EWPT strength.

However, the EWPT process depends on bosons and fermions. The boson gives a positive contribution (obey the Bose-Einstein distribution) but the fermion gives a negative contribution (obey the Fermi-Dirac distribution). In order to have the first order transition, the symmetry breaking process must generate mass for more bosons than fermions. 
TABLE VI. Values of the maximum of EWPT strength with $\omega=6 \mathrm{TeV}$.

\begin{tabular}{llcccc}
\hline \hline Period & Picture & $m_{Z_{2}}[\mathrm{TeV}]$ & $m_{N-R}[\mathrm{TeV}]$ & $S_{\text {Max }}$ without $N_{R}$ & $S_{\text {Max }}$ with $N_{R}$ \\
\hline$S U(3) \rightarrow S U(2)$ & $($ i $)$ & 2.386 & 2.227 & 70 & 50 \\
$S U(3) \rightarrow S U(2)$ & $($ ii $)$ & 2.254 & 1.986 & 100 & 30 \\
\hline \hline
\end{tabular}

In addition, in this model, the neutral fermion mass is generated from an effective operator. This operator which demonstrates an interaction between neutral fermions and two Higgs fields. The above neutral fermion is very different from usual fermions. The $M$ parameter in (48) has an energy dimension, and it may be an unknown dark interaction. Thus, the neutral fermions only are effective fermions, according to the Fermi-Dirac distribution, but their statistical nature needs to be further analyzed with other data.

\section{CONCLUSION AND OUTLOOKS}

In this paper, we have considered the EWPT in the 3-3-1-1 model where the SSB can be separated into two or three scales. Hence, in the model under consideration, the EWPT consists of two pictures. The first picture containing two periods of EWPT, has a transition $S U(3) \rightarrow S U(2)$ at $6 \mathrm{TeV}$ scale and another is $S U(2) \rightarrow U(1)$ transition which is the like-standard model EWPT. The second picture is an EWPT structure containing three periods, in which two first periods are similar to those of the first picture and another one is the symmetry breaking process of $U(1)_{N}$ subgroup. The EWPT is the first order phase transition if new bosons with mass within range of some $\mathrm{TeVs}$ are triggers for the purpose. The maximum strength of the $S U(2) \rightarrow U(1)$ phase transition is equal to 2.12 so the EWPT is not strong.

We have focused on the neutral fermions without lepton number being candidates for DM and obey the Fermi-Dirac distribution, and have shown that the mentioned fermions can be a negative trigger for EWPT. Furthermore, in order to be the strong first-order EWPT at $\mathrm{TeV}$ scale, the symmetry breaking processes must produce more bosons than fermions or the mass of bosons must be much larger than that of fermions.

It is known that the mass of Goldstone boson is very small [46] and the physical quantities are gauge independent so the critical temperature and the strength is gauge independent [19]. Consequently, the survey of effective potential in Landau gauge is also sufficient, or other word speaking, it is just consider in determined gauge. Thus, it is a reason why the Landau gauge is used in this work. In this paper, the structure of EWPT in the 3-3-1-1 model with the effective potential at finite temperature has been drawn at the 1-loop level; and this potential has two or three phases.

We have analyzed the processes which generate the masses for all gauge bosons inside the covariant derivatives. After diagonalization, the masses of gauge bosons do not have mixing among VEVs. Therefore, the EWPT stages are independent of each other [9].

To avoid higher (six) order Higgs self-interaction in the effective potential, the $f$ parameter associated with triple scalar antisymmetric coupling is ignored. Thus calculating the corrections with $f$ can reveal many new physical phenomena. In addition, from the phase transitions, we can get some bounds on the Higgs self-couplings.

In conclusion, the model has many bosons which will be good triggers for first-order EWPT. The situation is that as less heavy fermion as the result will be better. However, strength of EWPT can be reduced by many bosons (such as $Z, Z_{1}, Z_{2}$ in the 3-3-1-1 model).

The new scalar particles playing a role in generation mass for exotic particles, increase the value of EWPT strength. Because these scalar fields follow the BoseEinstein distribution, so that they contribute positively to the effective potential. With the help of such particles, the strength of phase transition will be strong. As mentioned above, their masses depend just on one VEV, so they only participate in one phase transition. Moreover, among the neutral fermions, they may be candidates for DM. From the point of view of the early universe, the above particles can be an inflaton or some product of the inflaton decay.

Although we only work on the 3-3-1-1 model, this manipulation can still apply to other models with multiperiod EWPT. We find that the results about bosons in Ref. [22] or new models (with $S U(5)$ or $S U(6)$ groups) in Ref. [21], can be a benchmark or may contain new material for the problem considered here-triggers for EWPT.

The heavy neutrinos or quarks mixing, in Ref. [3], are an interesting issue, and they may be the source for $C P$ violations. In order to analysis in detail baryogenesis, our next works will consider $C P$ violations and correction of neutral fermion-dark matter.

The model under consideration is an extension of the SM symmetry group, so it is renormalizable and there are no Landau poles when choosing the appropriate parameters. Inflation and kinetic mixing effect via $\rho$ parameter have been performed in Refs. [27-29]. We will also perform one UV completion of this model without the $f$ term in the Higgs potential.

It is interesting to note that the bound ( $w>3.2 \mathrm{TeV})$ obtained here from the EWPT is consistent with those followed from the oblique corrections in Ref. [30].

The largest cutoff of this model is $\Lambda$, may not be option. In addition, energy scale of the model goes from high to 
low $(\Lambda \rightarrow \omega \rightarrow u \sim v)$ so that the model has two cutoff scales which larger than $246 \mathrm{GeV}$. This is a common thing of all beyond SM.

We see that this model is correct for the $246 \mathrm{GeV}$ energy scale; the model has materials for the first order EWPT. However, this does not confirm that the model is correct at arbitrary energy level which requires further study/experimentation.

We also recognize that phases occur at different energy scales. The UV completion from the low to high scale, has been not clearly linked. In order to construct model, we need to consider EWPT, because the EWPT will make the appearance of UV completion. Therefore, in the next work with 3-3-1-1 model revisited, we will correct the model in combining with the UV completion for the Higgs potential as in Ref. [47].

\section{ACKNOWLEDGMENTS}

This research is funded by Vietnam National Foundation for Science and Technology Development (NAFOSTED) under Grant No. 103.01-2017.356.
[1] A. D. Sakharov, JETP Lett. 5, 24 (1967).

[2] K. Kajantie, M. Laine, K. Rummukainen, and M. Shaposhnikov, Phys. Rev. Lett. 77, 2887 (1996); F. Csikor, Z. Fodor, and J. Heitger, Phys. Rev. Lett. 82, 21 (1999); J. Grant and M. Hindmarsh, Phys. Rev. D 64, 016002 (2001); M. D'Onofrio, K. Rummukainen, and A. Tranberg, J. High Energy Phys. 08 (2012) 123.

[3] R. M. Fonseca and M. Hirsch, J. High Energy Phys. 08 (2016) 003.

[4] M. Bastero-Gil, C. Hugonie, S. F. King, D. P. Roy, and S. Vempati, Phys. Lett. B 489, 359 (2000); A. Menon, D. E. Morrissey, and C. E. M. Wagner, Phys. Rev. D 70, 035005 (2004); S. W. Ham, S. K. Oh, C. M. Kim, E. J. Yoo, and D. Son, Phys. Rev. D 70, 075001 (2004).

[5] J. M. Cline, G. Laporte, H. Yamashita, and S. Kraml, J. High Energy Phys. 07 (2009) 040.

[6] S. Kanemura, Y. Okada, and E. Senaha, Phys. Lett. B 606, 361 (2005); G. C. Dorsch, S. J. Huber, and J. M. No, J. High Energy Phys. 10 (2013) 029.

[7] S. W. Ham, S-A Shim, and S. K. Oh, Phys. Rev. D 81, 055015 (2010).

[8] V. Q. Phong, V. T. Van, and H. N. Long, Phys. Rev. D 88, 096009 (2013); V. Q. Phong, H. N. Long, V. T. Van, and N. C. Thanh, Phys. Rev. D 90, 085019 (2014); J. S. Borges and R. O. Ramos, Eur. Phys. J. C 76, 344 (2016).

[9] V. Q. Phong, H. N. Long, V. T. Van, and L. H. Minh, Eur. Phys. J. C 75, 342 (2015).

[10] C. W. Chiang, A. L. Kuo, and T. Yamada, J. High Energy Phys. 01 (2016) 120; C. W. Chiang and T. Yamada, Phys. Lett. B 06, 048 (2014); C. W. Chiang, A. L. Kuo, and T. Yamada, Phys. Rev. D 85, 095023 (2012).

[11] V. Q. Phong, N. C. Thao, and H. N. Long, Phys. Rev. D 97, 115008 (2018).

[12] J. R. Espinosa, T. Konstandin, and F. Riva, Nucl. Phys. B854, 592 (2012).

[13] S. Kanemura, E. Senaha, T. Shindou, and T. Yamada, J. High Energy Phys. 05 (2013) 066.

[14] D. J. H. Chung and A. J. Long, Phys. Rev. D 81, 123531 (2010).

[15] G. Barenboim and N. Rius, Phys. Rev. D 58, 065010 (1998).

[16] S. Profumo, M. J. Ramsey-Musolf, and G. Shaughnessy, J. High Energy Phys. 08 (2007) 010; S. Profumo, M. J.
Ramsey-Musolf, C. L. Wainwright, and P. Winslow, Phys. Rev. D 91, 035018 (2015); D. Curtin, P. Meade, and C-T. Yu, J. High Energy Phys. 11 (2014) 127; M. Jiang, L. Bian, W. Huang, and J. Shu, Phys. Rev. D 93, 065032 (2016).

[17] M. Carena, G. Nardini, M. Quiros, and C. E. M. Wagner, Nucl. Phys. B812, 243 (2009); A. Katz, M. Perelstein, M. J. Ramsey-Musolf, and P. Winslow, Phys. Rev. D 92, 095019 (2015); J. Kozaczuk, S. Profumo, L. S. Haskins, and C. L. Wainwright, J. High Energy Phys. 01 (2015) 144.

[18] H. H. Patel and M. J. Ramsey-Musolf, Phys. Rev. D 88, 035013 (2013); N. Blinov, J. Kozaczuk, D. E. Morrissey, and C. Tamarit, Phys. Rev. D 92, 035012 (2015); S. Inoue, G. Ovanesyan, and M. J. Ramsey-Musolf, Phys. Rev. D 93, 015013 (2016).

[19] H. H. Patel and M. J. Ramsey-Musolf, J. High Energy Phys. 07 (2011) 029; G. W. Anderson and L. J. Hall, Phys. Rev. D 45, 2685 (1992).

[20] S. Das, P. J. Fox, A. Kumar, and N. Weiner, J. High Energy Phys. 11 (2010) 108; D. Chung and A. J. Long, Phys. Rev. D 84, 103513 (2011); M. Carena, N. R. Shaha, and C. E. M. Wagner, Phys. Rev. D 85, 036003 (2012); A. Ahriche and S. Nasri, Phys. Rev. D 85, 093007 (2012); D. Borah and J. M. Cline, Phys. Rev. D 86, 055001 (2012).

[21] F. F. Deppisch, C. Hati, S. Patra, U. Sarkar, and J. W. F. Valle, Phys. Lett. B 762, 432 (2016).

[22] C. Furey, Eur. Phys. J. C 78, 375 (2018).

[23] F. Pisano and V. Pleitez, Phys. Rev. D 46, 410 (1992); P. H. Frampton, Phys. Rev. Lett. 69, 2889 (1992); R. Foot, O. F. Hernández, F. Pisano, and V. Pleitez, Phys. Rev. D 47, 4158 (1993).

[24] M. Singer, J. W. F. Valle, and J. Schechter, Phys. Rev. D 22, 738 (1980); R. Foot, H. N. Long, and T. A. Tran, Phys. Rev. D 50, R34 (1994); J. C. Montero, F. Pisano, and V. Pleitez, Phys. Rev. D 47, 2918 (1993); H. N. Long, Phys. Rev. D 54, 4691 (1996); 53, 437 (1996).

[25] A. E. C. Hernández, S. Kovalenko, H. N. Long, and I. Schmidt, J. High Energy Phys. 07 (2018) 144.

[26] J. G. Ferreira, C. A de S. Pires, J. G. Rodrigues, and P. S. R. da Silva, Phys. Lett. B 771, 199 (2017).

[27] P. V. Dong, H. T. Hung, and T. D. Tham, Phys. Rev. D 87, 115003 (2013). 
[28] P. V. Dong, D. T. Huong, F. S. Queiroz, and N. T. Thuy, Phys. Rev. D 90, 075021 (2014).

[29] D. T. Huong, P. V. Dong, C. S. Kim, and N. T. Thuy, Phys. Rev. D 91, 055023 (2015).

[30] P. V. Dong and D. T. Si, Phys. Rev. D 93, 115003 (2016).

[31] D. T. Huong and P. V. Dong, Eur. Phys. J. C77, 204 (2017).

[32] P. V. Dong, D. T. Huong, D. A. Camargo, F. S. Queiroz, and J. W. F. Valle, arXiv:1805.08251.

[33] D. Chang and H. N. Long, Phys. Rev. D 73, 053006 (2006).

[34] P. V. Dong, Phys. Rev. D 92, 055026 (2015).

[35] M. E. Peskin and T. Takeuchi, Phys. Rev. D 46, 381 (1992).

[36] G. Cacciapaglia, C. Csaki, G. Marandella, and A. Strumia, Phys. Rev. D 74, 033011 (2006); G. Funk, D. O'Neil, and R. M. Winters, Int. J. Mod. Phys. A 27, 1250021 (2012).

[37] K. Sasaki, Phys. Lett. B 308, 297 (1993); P. H. Frampton and M. Harada, Phys. Rev. 58, 095013 (1998); H. N. Long and T. Inami, Phys. Rev. D 61, 075002 (2000).

[38] C. Salazar, R. H. Benavides, W. A. Ponce, and E. Rojas, J. High Energy Phys. 07 (2015) 096.
[39] A. E. C. Hernandez, R. Martinez, and F. Ochoa, Phys. Rev. D 73, 035007 (2006).

[40] R. Martinez and F. Ochoa, Phys. Rev. D 77, 065012 (2008).

[41] A. J. Buras, F. De Fazio, and J. Girrbach, J. High Energy Phys. 02 (2014) 112.

[42] A. J. Buras, F. De Fazio, and J. Girrbach-Noe, J. High Energy Phys. 08 (2014) 039.

[43] A. J. Buras, F. De Fazio, J. Girrbach, and M. V. Carlucci, J. High Energy Phys. 02 (2013) 023.

[44] M. J. Baker, M. Breitbach, J. Kopp, and L. Mittnacht, J. High Energy Phys. 03 (2018) 114.

[45] D. A. Gutierrez, W. A. Ponce, and L. A. Sanchez, Eur. Phys. J. C 46, 497 (2006); Y. A. Coutinho, V. S. Guimaraes, and A. A. Nepomuceno, Phys. Rev. D 87, 115014 (2013).

[46] C. Patrignani et al. (Particle Data Group), Chin. Phys. C 40, 100001 (2016).

[47] A. Agugliaro, O. Antipin, D. Becciolini, S. De Curtis, and M. Redi, Phys. Rev. D 95, 035019 (2017); F. Caracciolo, A. Parolini, and M. Serone, J. High Energy Phys. 02 (2013) 066. 